\title{
Modeling convection in solidification processes using stabilized finite element techniques
}

\author{
Deep Samanta and Nicholas Zabaras*† \\ Materials Process Design and Control Laboratory, Sibley School of Mechanical and Aerospace Engineering, \\ 188 Frank H. T. Rhodes Hall, Cornell University, \\ Ithaca, NY 14853-3801, U.S.A
}

\begin{abstract}
SUMMARY
Solidification of dendritic alloys is modeled using stabilized finite element techniques to study convection and macrosegregation driven by buoyancy and shrinkage. The adopted governing macroscopic conservation equations of momentum, energy and species transport are derived from their microscopic counterparts using the volume-averaging method. A single domain model is considered with a fixed numerical grid and without boundary conditions applied explicitly on the freezing front. The mushy zone is modeled here as a porous medium with either an isotropic or an anisotropic permeability. The stabilized finite-element scheme, previously developed by authors for modeling flows with phase change, is extended here to include effects of shrinkage, density changes and anisotropic permeability during solidification. The fluid flow scheme developed includes SUPG (streamline-upwind/Petrov-Galerkin), PSPG (pressure stabilizing/Petrov-Galerkin), DSPG (Darcy stabilizing/Petrov-Galerkin) and other stabilizing terms arising from changes in density in the mushy zone. For the energy and species equations a classical SUPG-based finite element method is employed with minor modifications. The developed algorithms are first tested for a reference problem involving solidification of Lead-Tin alloy where the mushy zone is characterized by an isotropic permeability. Convergence studies are performed to validate the simulation results. Solidification of the same alloy in the absence of shrinkage is studied to observe differences in macrosegregation. Vertical solidification of a Lead-Tin alloy, where the mushy zone is characterized by an anisotropic permeability, is then simulated. The main aim here is to study convection and demonstrate formation of freckles and channels due to macrosegregation. The ability of stabilized finite element methods to model a wide variety of solidification problems with varying underlying phenomena in two- and three-dimensions is demonstrated through these examples. Copyright (c) 2005 John Wiley \& Sons, Ltd.
\end{abstract}

KEY WORDS: Solidification; stabilized finite element method; convection; shrinkage; density change; anisotropic permeability

${ }^{*}$ Correspondence to: Nicholas Zabaras, Materials Process Design and Control Laboratory, Sibley School of Mechanical and Aerospace Engineering, 188 Frank H. T. Rhodes Hall, Cornell University, Ithaca, NY 148533801, U.S.A

†Email: zabaras@cornell.edu

Contract/grant sponsor: University-Industry Partnerships for Aluminum Industry of the Future Program of the Office of Industrial Technologies of the U.S. Department of Energy; contract/grant number: DE-FC0702ID14396 


\section{INTRODUCTION}

Solidification of alloys invariably results in the formation of a two-phase mushy zone. This zone, where solid and liquid phases coexist, plays an important role in determining the final properties of a cast alloy. Most solidifying alloys are accompanied by macrosegregation, i.e. large scale non-uniformity of local solute composition. Macrosegregation leads to a wide variation of properties in the final cast structure and is therefore a leading cause of defects in alloys. Inter-dendritic melt flow caused by thermal and solutal buoyancy forces and shrinkage in the mushy zone, which arises because of density differences between the solid and liquid phases, is one of the leading causes of macrosegregation. Shrinkage in the solidifying alloy caused by this volume change results in the casting being pulled away from the mold wall and induces defects such as hot tears and porosity. Single domain continuum models have been used extensively [1]-[3] to simulate solidification of binary alloys. Xu and $\mathrm{Li}$ in $[4,5]$ were the first to use a continuum model to simulate solidification of an alloy incorporating the effects of density changes. In their simulations, the mushy zone was characterized by an isotropic permeability. One of their primary observations was that a large pressure gradient is required in the mushy zone so that feed liquid can compensate for volume changes occurring during solidification. Shrinkage induced flows were found to dominate in regions at or near the solidus temperature. Heinrich and co-workers in [6]-[9] have modeled solidification of Lead-Tin alloys where the mushy zone is characterized by an anisotropic permeability. In [8], Heinrich et al. used finite element methods to model flow driven by density changes during phase change. They discretized the governing equation for fluid flow using the penalty formulation along with the classical SUPG method. Incropera and Krane in [10] analyzed the effect of shrinkage on macrosegregation during the solidification of a Lead-Tin alloy. They employed a continuum model based on the mixture theory for their analysis and used finite difference methods to solve the governing transport equations.

Over the past few years finite element methods have been increasingly used for modeling complex alloy solidification problems that were previously tackled by finite difference and finite volume solution methodologies. Stabilized finite element methods, characterized by their superior convergence rates, have gained greater acceptability in recent years due to vast improvements in computational power and capability. In [11], the authors had proposed a stabilized finite element method for analyzing flow in porous media and binary alloy solidification problems where convection was dominant. The mushy zone was characterized by an isotropic permeability for all two-dimensional examples considered in [11]. Building up on our earlier work, we extend the stabilized finite element method to model solidification of alloys where both buoyancy and shrinkage driven flows exist and where permeability of the mushy zone can be either isotropic or anisotropic. The developed methodology is general in nature and is applicable for a wide range of problems. Stabilized finite element methods have matured over the past few years and their ability in modeling a wide class of solidification problems with diverse underlying phenomena in both two- and three-dimensions is demonstrated in this work through various numerical examples.

The organization of the paper is as follows. In Section 2, the mathematical model, used for simulating solidification of alloys with different but constant phase densities, is presented. This model is derived from microscopic conservation equations and the details of the derivation process are not repeated here. The reader is referred to [12]-[14] for more details. To complete the model, closure relationships, like those derived from thermodynamic relations for phase 


\begin{tabular}{|c|c|c|c|}
\hline \multicolumn{4}{|c|}{ NOMENCLATURE } \\
\hline $\mathbf{A}$ & assembly over elements & $\mu$ & fluid viscosity \\
\hline$C$ & solute concentration & $\nu$ & kinematic viscosity \\
\hline$c$ & specific heat & $\rho$ & density \\
\hline$D$ & mass diffusivity & $\Omega$ & problem domain \\
\hline$d$ & dendrite arm spacing & $\tau$ & stabilizing parameters \\
\hline e & direction cosines & $\zeta$ & viscous stabilizing term \\
\hline$f$ & liquid mass fraction & & \\
\hline$G$ & vertical thermal gradient & \multicolumn{2}{|l|}{ Subscripts } \\
\hline$g$ & gravity & $a m b$ & ambient \\
\hline \multirow[t]{2}{*}{$h$} & enthalpy, convection & $C$ & solutal \\
\hline & heat transfer coefficient & $\operatorname{conv}$ & convection \\
\hline$K$ & permeability & eut & eutectic \\
\hline$k$ & thermal conductivity & $f$ & fusion \\
\hline$m$ & slope of phase diagram lines & $g$ & gravity \\
\hline$p$ & pressure & $i$ & initial \\
\hline$r$ & cooling rate & $l$ & liquid \\
\hline$R e$ & Reynolds number & $\operatorname{liq}$ & liquidus \\
\hline$t$ & time & 10 & liquid reference \\
\hline$T$ & temperature & $m$ & melting \\
\hline$v$ & velocity vector & $\max$ & maximum \\
\hline$W, V$ & function spaces & $\min$ & minimum \\
\hline$|v|$ & velocity magnitude & $\mathrm{n}, \mathrm{n}+1$ & time step index \\
\hline $\boldsymbol{x}$ & position vector & 0 & reference/initial \\
\hline$x$ & $\mathrm{x}$ coordinate & its & solid \\
\hline$y$ & y coordinate & sol & solidus \\
\hline \multirow[t]{2}{*}{$z$} & $\mathrm{z}$ coordinate & $T$ & thermal \\
\hline & & tol & tolerance \\
\hline \multicolumn{4}{|c|}{ Greek symbols } \\
\hline$\alpha$ & thermal diffusivity & Superscripts & \\
\hline$\beta$ & coefficient of expansion & $e$ & element domain \\
\hline$\Delta_{v}$ & change in volume & $h$ & finite element \\
\hline$\delta$ & advection stabilizing term & & discretization \\
\hline$\epsilon$ & liquid volume fraction & $\mathrm{j}, \mathrm{j}+1$ & iteration index \\
\hline$\eta$ & pressure stabilizing term & $n_{e l}$ & number of elements \\
\hline$\gamma$ & Darcy stabilizing term & $T$ & transpose \\
\hline$\kappa_{p}$ & partition coefficient & & \\
\hline
\end{tabular}


change, are used. A detailed description of these relationships is given in Section 2. In Section 3, the stabilized finite element formulation for the fluid-flow problem is presented along with a brief discussion of various stabilizing terms. In addition to those present in the model developed in [11], extra stabilizing terms, arising from density changes in the mushy zone, are described here. Discretization of energy and species equations is briefly described in Section 4, which is followed by a brief description of the overall solution algorithm and solution techniques in 5. Numerical examples to validate this method are discussed in Section 6. Solidification of a Lead-Tin alloy under the influence of convection driven by buoyancy and shrinkage is modeled first. The results obtained are compared with those given in [10]. Convergence studies are also performed by refining the finite element mesh. The developing mushy zone is assumed to be a porous medium of isotropic permeability. The problem is then repeated after assuming equal phase densities, which eliminates the effect of shrinkage. Differences in macrosegregation are observed for both cases, and this highlights differences in the underlying phenomenon. Vertical solidification of a Lead-Tin alloy, where the mushy zone is characterized by an anisotropic permeability, is considered next. In this example, the development of channels and freckles is observed. Macrosegregation in a solidifying alloy under the influence of convection is studied in all these examples. Convection is either driven by the combined influence of thermosolutal buoyancy and shrinkage or by thermosolutal buoyancy alone.

Box I : Governing equations for solidification of alloys with different phase densities

$$
\begin{gathered}
\frac{\partial \rho(\boldsymbol{x}, t)}{\partial t}+\nabla \cdot(\rho(\boldsymbol{x}, t) \boldsymbol{v}(\boldsymbol{x}, t))=0, \quad \boldsymbol{x} \in \Omega \\
\frac{\partial(\rho(\boldsymbol{x}, t) \boldsymbol{v}(\boldsymbol{x}, t))}{\partial t}+\nabla \cdot\left(\frac{\rho(\boldsymbol{x}, t) \boldsymbol{v}(\boldsymbol{x}, t) \boldsymbol{v}(\boldsymbol{x}, t)}{f(\boldsymbol{x}, t)}\right)=-\nabla p(\boldsymbol{x}, t)+\frac{p(\boldsymbol{x}, t)}{\epsilon(\boldsymbol{x}, t)} \nabla \epsilon(\boldsymbol{x}, t) \\
+\nabla \cdot\left[\frac{\mu}{\rho_{l}}\left(\nabla(\rho(\boldsymbol{x}, t) \boldsymbol{v}(\boldsymbol{x}, t))+(\nabla(\rho(\boldsymbol{x}, t) \boldsymbol{v}(\boldsymbol{x}, t)))^{T}\right)\right]-\frac{\epsilon \mu}{K(\epsilon)} \frac{\rho(\boldsymbol{x}, t)}{\rho_{l}} \boldsymbol{v}(\boldsymbol{x}, t) \\
-\epsilon(\boldsymbol{x}, t) \rho_{l_{0}} g\left[\beta_{T}\left(T(\boldsymbol{x}, t)-T_{0}\right)+\beta_{s}\left(C_{l}(\boldsymbol{x}, t)-C_{l_{0}}\right)\right] \boldsymbol{e}_{\boldsymbol{g}}, \quad \boldsymbol{x} \in \Omega \\
\frac{\partial(\rho(\boldsymbol{x}, t) h(\boldsymbol{x}, t))}{\partial t}+\nabla \cdot\left(\rho(\boldsymbol{x}, t) \boldsymbol{v}(\boldsymbol{x}, t) h_{l}(\boldsymbol{x}, t)\right)=\nabla \cdot\left[\left(\epsilon(\boldsymbol{x}, t) k_{l}+(1-\epsilon(\boldsymbol{x}, t)) k_{s}\right) \nabla T(\boldsymbol{x}, t)\right], \quad \boldsymbol{x} \in \Omega \\
\frac{\partial(\rho(\boldsymbol{x}, t) C(\boldsymbol{x}, t))}{\partial t}+\nabla \cdot\left(\rho(\boldsymbol{x}, t) \boldsymbol{v}(\boldsymbol{x}, t) C_{l}(\boldsymbol{x}, t)\right)=\nabla \cdot\left(\rho(\boldsymbol{x}, t) f(\boldsymbol{x}, t) D_{l} \nabla C_{l}(\boldsymbol{x}, t)\right), \quad \boldsymbol{x} \in \Omega
\end{gathered}
$$

Initial conditions:

$$
\boldsymbol{v}(\boldsymbol{x}, 0)=\mathbf{0}, \quad h(\boldsymbol{x}, 0)=h_{i}, \quad C(\boldsymbol{x}, 0)=C_{i}, \quad \rho(\boldsymbol{x}, 0)=\rho_{l_{0}}, \quad \frac{\rho(\boldsymbol{x}, 0)}{\rho_{l}(\boldsymbol{x}, 0)}=1.0 \quad \boldsymbol{x} \in \Omega
$$

\section{MATHEMATICAL MODEL FOR ALLOY SOLIDIFICATION}

Governing transport equations for solidification of alloys are summarized in Box I. These are obtained by volume averaging the microscopic transport equations as described in [12]-[14]. We 


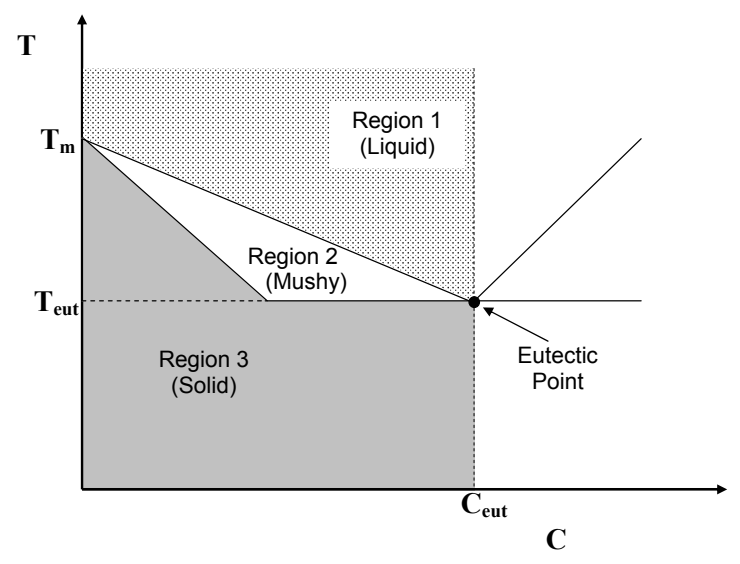

Figure 1. The phase diagram for a binary alloy. Individual values are given in Table I.

assume that there are only two phases and no pore formation occurs. The liquid is assumed to be Newtonian, the flow laminar and the solid phase stationary. We also assume conservation of both mass and volume. $\epsilon$ denotes the liquid volume fraction and $f$ the liquid mass fraction. With the assumption of a linear lever-rule model, $f$ is calculated from the phase diagram. $\epsilon$ can then be calculated as $\epsilon=\rho f / \rho_{l}$. The mushy zone is modeled as a porous medium characterized by either an isotropic or anisotropic permeability. The former is given by the Kozeny-Carman relationship as follows:

$$
K(\epsilon)=\frac{K_{0} \epsilon^{3}}{(1-\epsilon)^{2}}
$$

where $K_{0}$ is a permeability constant that depends on the morphology of the two-phase mushy region as $K_{0}=d^{2} / 180$, with $d$ denoting the secondary dendrite arm spacing. When the permeability is assumed to be anisotropic, it is expressed in principal growth directions by

$$
\begin{gathered}
K_{x}(\epsilon)=K_{y}(\epsilon)=\left\{\begin{array}{cc}
1.09 \times 10^{-3} \epsilon^{3.32} d^{2} & \epsilon \leq 0.65 \\
4.04 \times 10^{-6}[\epsilon /(1-\epsilon)]^{6.7336} d^{2} & 0.65<\epsilon \leq 0.75 \\
\left(-6.49 \times 10^{-2}+5.43 \times 10^{-2}[\epsilon /(1-\epsilon)]^{0.25}\right) d^{2} & 0.75<\epsilon<1.0
\end{array}\right. \\
K_{z}(\epsilon)=\left\{\begin{array}{cc}
3.75 \times 10^{-4} \epsilon^{2} d^{2} & \epsilon \leq 0.65 \\
2.05 \times 10^{-7}[\epsilon /(1-\epsilon)]^{10.739} d^{2} & 0.65<\epsilon \leq 0.75 \\
0.074\left[\log (1-\epsilon)^{-1}-1.49+2(1-\epsilon)-0.5(1-\epsilon)^{2}\right] d^{2} & 0.75<\epsilon<1.0
\end{array}\right.
\end{gathered}
$$

Equations (7) and (8) express permeability in directions transverse and parallel to the dendrite growth, respectively. These relations are listed in [6]-[9] and have been obtained from experimental data through curve fit and regression analysis.

Closure relationships for variables like temperature, solute concentrations and mass fractions of individual phases are necessary to complete the description of the mathematical model. The closure relationships described in this section are similar to those given in $[1,2,11]$. With the assumption of thermal equilibrium of all phases in the averaging volume, we have $T_{l}=T_{s}=T$. 
Enthalpies of the liquid and solid phases are given by,

$$
\begin{aligned}
h_{l} & =c_{l} T+h_{l 0} \\
h_{s} & =c_{s} T
\end{aligned}
$$

where $h_{l 0}$ is the reference enthalpy expressed as

$$
h_{l 0}=\left(c_{s}-c_{l}\right) T_{\text {eut }}+h_{f}
$$

and $h_{f}$ is the enthalpy of fusion at the eutectic temperature $T_{\text {eut }}$. Using Equations (9), (10) and (11), the mixture enthalpy is expressed in terms of temperature as:

$$
h=f h_{l}+(1-f) h_{s}=f\left[\left(c_{l}-c_{s}\right)\left(T-T_{\text {eut }}\right)+h_{f}\right]+c_{s} T
$$

We use the phase diagram, shown in Fig. 1, for our alloy solidification model with the partition coefficient, $\kappa_{p}=m_{l i q} / m_{\text {sol }}$ being a constant. $m_{l i q}$ and $m_{\text {sol }}$ denote the slopes of liquidus and solidus lines, respectively. The local conservation of concentration in terms of the solid and liquid solute concentrations is expressed as

$$
C=f C_{l}+(1-f) C_{s}
$$

With the assumption of equilibrium, the solid concentration is related to the liquid concentration through the equilibrium partition ratio $\kappa_{p}=C_{s} / C_{l}$. The liquid solute concentration, after neglecting under-cooling, is related to the temperature as:

$$
C_{l i q}=\frac{T-T_{m}}{m_{l i q}}
$$

When the densities of the two phases are not equal, $\rho_{l} \neq \rho_{s}$, the mixture density is expressed as

$$
\rho=\epsilon \rho_{l}+(1-\epsilon) \rho_{s}
$$

Assuming conservation of volume, we have $\epsilon_{l}+\epsilon_{s}=1$. This yields

$$
\rho\left(\frac{f}{\rho_{l}}+\frac{1-f}{\rho_{s}}\right)=1
$$

Some relevant variables, required in update formulae for temperature $T$, liquid solute concentration $C_{l}$ and liquid volume fraction $\epsilon$, are defined as follows:

$$
\begin{aligned}
T_{\text {liq }} & =T_{m}+m_{\text {liq }} C \\
T_{\text {sol }} & =\max \left\{T_{m}+\frac{m_{\text {liq }}}{\kappa_{p}} C, T_{\text {eut }}\right\} \\
h_{\text {liq }} & =c_{l} T_{\text {liq }}+h_{l 0} \\
h_{\text {sol }} & =c_{s} T_{\text {sol }} \\
h_{e} & =f_{\text {eut }} h_{f}+c_{s} T_{\text {eut }}
\end{aligned}
$$

With the assumption of conservation of mass and volume, the mixture density $\rho$ can be calculated from the liquid mass fraction $f$. The Lever rule is used to calculate the liquid mass fraction and is given by

$$
f=1-\frac{1}{1-\kappa_{p}}\left(\frac{T-T_{l i q}}{T-T_{m}}\right)
$$

Update formulae for various thermodynamic variables are listed below: 
- When $h>h_{l i q}$, the particular point is occupied by pure liquid, corresponding to region (1) in Fig. 1. The following holds: $T=\frac{h-h_{l 0}}{c_{l}}, f=1, \epsilon=1$ and $C_{l}=C$

- When $h_{e}<h \leq h_{\text {liq }}$, solidification has started but the liquid concentration has not reached the eutectic point, corresponding to region (2) in Fig. 1. In this region, the liquid mass fraction, $f$, and temperature, $T$, are solved iteratively using $T=\frac{h-f h_{l 0}}{f c_{l}+(1-f) c_{s}}$ and $f=\left[1-\frac{1}{1-k_{p}}\left(\frac{T-T_{l i q}}{T-T_{m}}\right)\right]$. Once $T$ and $f$ converge, $C_{l}$ and $\rho$ are calculated from Equations (14) and (16), respectively. The liquid volume fraction, $\epsilon$, is calculated as $\epsilon=\rho f / \rho_{l}$.

- When $h_{\text {sol }}<h \leq h_{e}$, the melt in the control volume is solidified at eutectic temperature and eutectic concentration. In this case, $T=T_{\text {eut }}$ and $C_{l}=C_{\text {eut }} . f$ is calculated from Equation (12) as $f=\frac{h-h_{\text {sol }}}{h_{f}}$. Both $\rho$ and $\epsilon$ are calculated from $f$.

- When $h \leq h_{\text {sol }}$, the alloy in the averaging volume is completely solidified, corresponding to region (3) in Fig. 1. In this case, $T=\frac{h}{c_{s}}, C_{s}=C$ and $\rho=\rho_{s}$. Both $f$ and $\epsilon$ are zero.

\section{STABILIZED FINITE ELEMENT METHOD FOR FLUID FLOW}

Let us define the function spaces $S_{\boldsymbol{v}}$ and $S_{p}$ as follows:

$$
\begin{aligned}
S_{\boldsymbol{v}} \stackrel{\text { def }}{=} & \left\{\boldsymbol{v} \mid \boldsymbol{v} \in\left(L_{2}(\Omega)\right)^{\mathrm{nsd}}, \operatorname{div} \boldsymbol{v} \in L_{2}(\Omega), \boldsymbol{v}=0 \text { on } \Gamma\right\} \\
S_{p} \stackrel{\text { def }}{=} & \left\{p \mid p \in L_{2}(\Omega), \int_{\Omega} p d \Omega=0\right\}
\end{aligned}
$$

The classical Galerkin formulation for the flow problem, given by Equations (1) and (2) in Box I, can be stated as follows: Find $\boldsymbol{V} \stackrel{\text { def }}{=}\{\boldsymbol{v}, p\} \in S \boldsymbol{v} \times S_{p}$ such that for all $\boldsymbol{W} \stackrel{\text { def }}{=}\{\boldsymbol{w}, q\} \in S \boldsymbol{v} \times S_{p}$ the following holds:

$$
B(\boldsymbol{W}, \boldsymbol{V})=L(\boldsymbol{W})
$$

where

$$
\begin{aligned}
B(\boldsymbol{W}, \boldsymbol{V}) & =\int_{\Omega} \boldsymbol{w} \cdot\left(\frac{\rho \partial \boldsymbol{v}}{\partial t}+\frac{\rho \boldsymbol{v} \cdot \nabla \boldsymbol{v}}{f}-\frac{\partial \rho}{\partial t} \frac{(1-f) \boldsymbol{v}}{f}+\frac{\epsilon \mu \rho}{K(\epsilon) \rho_{l}} \boldsymbol{v}\right) d \Omega \\
& -\int_{\Omega} p \nabla \cdot \boldsymbol{w} d \Omega+\int_{\Omega} \frac{\mu \rho}{\rho_{l}} \nabla \boldsymbol{w} \cdot\left(\nabla \boldsymbol{v}+(\nabla \boldsymbol{v})^{T}\right) d \Omega \\
& +\int_{\Omega} \frac{\mu}{\rho_{l}} \nabla \boldsymbol{w} \cdot\left(\boldsymbol{v} \otimes \nabla \rho+(\boldsymbol{v} \otimes \nabla \rho)^{T}\right) d \Omega \\
& +\int_{\Omega} q \rho \nabla \cdot \boldsymbol{v} d \Omega+\int_{\Omega} q \boldsymbol{v} \cdot \nabla \rho d \Omega \\
L(\boldsymbol{W}) & =\int_{\Omega} \frac{p}{\epsilon} \boldsymbol{w} \cdot \nabla \epsilon d \Omega-\int_{\Omega} \boldsymbol{w} \cdot \epsilon \rho_{l_{0}} g\left(\beta_{T}\left(T-T_{0}\right)+\beta_{C}\left(C_{l}-C_{l_{0}}\right)\right) \boldsymbol{e}_{\boldsymbol{g}} d \Omega \\
& -\int_{\Omega} q \frac{\partial \rho}{\partial t} d \Omega
\end{aligned}
$$

Terms involving gradient of density, $\nabla \rho$, arise due to varying densities in the mushy zone. In introducing the FEM used here, let us first define a modified pressure space $S_{p}^{\prime}$ as follows:

$$
S_{p}^{\prime} \stackrel{\text { def }}{=}\left\{p \mid p \in H^{1}(\Omega), \int_{\Omega} q d \Omega=0\right\}
$$


The stabilized weak form proposed here is the following: Find $\boldsymbol{V}=\{\boldsymbol{v}, p\} \in S_{\boldsymbol{v}} \times S_{p}^{\prime}$ such that for all $\boldsymbol{W}=\{\boldsymbol{w}, q\} \in S_{\boldsymbol{v}} \times S_{p}^{\prime}$ the following holds:

$$
B_{\text {stab }}(\boldsymbol{W}, \boldsymbol{V})=L_{\mathrm{stab}}(\boldsymbol{W})
$$

where:

$$
\begin{gathered}
B_{\mathrm{stab}}(\boldsymbol{W}, \boldsymbol{V})=B(\boldsymbol{W}, \boldsymbol{V})+\int_{\Omega} \mathcal{F}(\boldsymbol{v}, p) \cdot \mathcal{G}(\boldsymbol{w}, q) d \Omega \\
L_{\mathrm{stab}}(\boldsymbol{W})=L(\boldsymbol{W})+\int_{\Omega}\left(\frac{p}{\epsilon} \nabla \epsilon-\epsilon \rho_{l_{0}} g\left(\beta_{T}\left(T-T_{0}\right)+\beta_{C}\left(C_{l}-C_{l_{0}}\right)\right) \boldsymbol{e}_{\boldsymbol{g}}\right) \cdot \mathcal{G}(\boldsymbol{w}, q) d \Omega
\end{gathered}
$$

where we defined:

$$
\begin{aligned}
\mathcal{F}(\boldsymbol{v}, p) & =\rho \frac{\partial \boldsymbol{v}}{\partial t}+\frac{\rho \boldsymbol{v}_{*} \cdot \nabla \boldsymbol{v}}{f}-\frac{\partial \rho}{\partial t} \frac{(1-f) \boldsymbol{v}}{f}+\nabla p+\frac{\epsilon \mu \rho}{K(\epsilon) \rho_{l}} \boldsymbol{v} \\
& -\nabla \cdot\left[\frac{\mu}{\rho_{l}}\left(\nabla(\rho \boldsymbol{v})+(\nabla(\rho \boldsymbol{v}))^{T}\right)\right] \\
\mathcal{G}(\boldsymbol{w}, q) & =\tau_{1} \frac{\boldsymbol{v}_{*} \cdot \nabla \boldsymbol{w}}{f}-\tau_{1} \frac{1}{\rho} \frac{\partial \rho}{\partial t} \frac{(1-f) \boldsymbol{w}}{f}-\tau_{2} \frac{\epsilon \mu}{K(\epsilon) \rho_{l}} \boldsymbol{w} \\
& -\frac{\tau_{3}}{\rho} \nabla \cdot\left[\frac{\mu}{\rho_{l}}\left(\nabla(\rho \boldsymbol{w})+(\nabla(\rho \boldsymbol{w}))^{T}\right)\right]+\tau_{4} \nabla q
\end{aligned}
$$

where $\boldsymbol{v}_{*}$ denotes the velocity obtained from the previous iteration.

The stabilizing terms in Equations (29) and (30) can be derived by various techniques including a least squares procedure. In addition to those discussed in [11], extra stabilizing terms arise due to change in density in the mushy zone. The sign of the Darcy term used here is the reverse of that obtained by least squares [15]. This sign reversal, first discussed in [15], was verified in our previous work in [11]. The particular values of the parameters $\tau_{1}, \ldots, \tau_{4}$, used in this work, are introduced later in this section.

Let us now consider a given finite element partition $\bar{\Omega}=\bigcup_{e=1}^{n_{e l}} \bar{\Omega}^{e}$. To avoid high sensitivity of the fluid flow simulator on the variation of $\epsilon$ or $f$, we neglect terms that involve gradients of these fields in the implementation of Equation (28). This leads to simplification of the final weak form of equations and helps in improving the convergence rate.

We next define, for the given finite element partition, spaces $S_{\boldsymbol{v}}^{h}$ and $S_{p}^{h^{\prime}}$ as follows:

$$
\begin{aligned}
S_{\boldsymbol{v}}^{h} \stackrel{\text { def }}{=} & \left\{\boldsymbol{v}^{h}\left|\boldsymbol{v}^{h} \in S_{v}, \boldsymbol{v}^{h} \in\left(C^{o}(\bar{\Omega})\right)^{n_{s d}}, \boldsymbol{v}^{h}\right|_{\Omega^{e}} \in\left(P\left(\Omega^{e}\right)\right)^{n_{s d}}, e=1,2, \ldots, n_{e l}\right\} \\
S_{p}^{h^{\prime}} \stackrel{\text { def }}{=} & \left\{p^{h}\left|p^{h} \in S_{p}, p^{h} \in C^{o}(\bar{\Omega}), p^{h}\right|_{\Omega^{e}} \in P\left(\Omega^{e}\right), e=1,2, \ldots, n_{e l}\right\}
\end{aligned}
$$

The final FEM is then posed as follows: Find $\boldsymbol{V}^{h}=\left\{\boldsymbol{v}^{h}, p^{h}\right\} \in S_{\boldsymbol{v}}^{h} \times S_{p}^{h^{\prime}}$ such that for all $\boldsymbol{W}^{h}=\left\{\boldsymbol{w}^{h}, q^{h}\right\} \in S_{\boldsymbol{v}}^{h} \times S_{p}^{h^{\prime}}$ the following holds:

$$
B_{\text {stab }}^{h}\left(\boldsymbol{W}^{h}, \boldsymbol{V}^{h}\right)=L_{\text {stab }}^{h}\left(\boldsymbol{W}^{h}\right)
$$

with

$$
B_{\mathrm{stab}}^{h}\left(\boldsymbol{W}^{h}, \boldsymbol{V}^{h}\right)=B^{h}\left(\boldsymbol{W}^{h}, \boldsymbol{V}^{h}\right)+\underset{e=1}{n_{e l}} \int_{\Omega^{e}} \mathcal{F}^{h}\left(\boldsymbol{v}^{h}, p^{h}\right) \cdot \mathcal{G}^{h}\left(\boldsymbol{w}^{h}, q^{h}\right) d \Omega^{e}
$$


$L_{\mathrm{stab}}\left(\boldsymbol{W}^{h}\right)=L\left(\boldsymbol{W}^{h}\right)+\underset{e=1}{n_{e l}} \int_{\Omega^{e}}-\epsilon \rho_{l_{0}} g\left(\beta_{T}\left(T^{h}-T_{0}\right)+\beta_{C}\left(C_{l}^{h}-C_{l_{0}}\right)\right) \boldsymbol{e}_{\boldsymbol{g}} \cdot \mathcal{G}^{h}\left(\boldsymbol{w}^{h}, q^{h}\right) d \Omega$

where we have defined $\mathcal{F}^{h}\left(\boldsymbol{v}^{h}, p^{h}\right)$ and $\mathcal{G}^{h}\left(\boldsymbol{w}^{h}, q^{h}\right)$ as:

$$
\begin{aligned}
\mathcal{F}\left(\boldsymbol{v}^{h}, p^{h}\right) & =\rho \frac{\partial \boldsymbol{v}^{h}}{\partial t}+\frac{\rho \boldsymbol{v}_{*} \cdot \nabla \boldsymbol{v}^{h}}{f}-\frac{\partial \rho}{\partial t} \frac{(1-f) \boldsymbol{v}^{h}}{f}+\nabla p^{h}+\frac{\epsilon \mu \rho}{K(\epsilon) \rho_{l}} \boldsymbol{v}^{h} \\
& -\nabla \cdot\left[\frac{\mu}{\rho_{l}}\left(\nabla\left(\rho \boldsymbol{v}^{h}\right)+\left(\nabla\left(\rho \boldsymbol{v}^{h}\right)\right)^{T}\right)\right] \\
\mathcal{G}(\boldsymbol{w}, q) & =\tau_{1} \frac{\boldsymbol{v}_{*} \cdot \nabla \boldsymbol{w}^{h}}{f}-\tau_{1} \frac{1}{\rho} \frac{\partial \rho}{\partial t} \frac{(1-f) \boldsymbol{w}^{h}}{f}-\tau_{2} \frac{\epsilon \mu}{K(\epsilon) \rho_{l}} \boldsymbol{w}^{h} \\
& -\frac{\tau_{3}}{\rho} \nabla \cdot\left[\frac{\mu}{\rho_{l}}\left(\nabla\left(\rho \boldsymbol{w}^{h}\right)+\left(\nabla\left(\rho \boldsymbol{w}^{h}\right)\right)^{T}\right)\right]+\tau_{4} \nabla q^{h}
\end{aligned}
$$

The stabilizing contributions from the advective, Darcy, viscous and pressure terms are expressed as:
(A) $\quad \delta^{h}=\tau_{1}^{e} \frac{\boldsymbol{v}_{*}^{h} \cdot \nabla \boldsymbol{w}^{h}}{f}-\tau_{1}^{e} \frac{1}{\rho} \frac{\partial \rho}{\partial t} \frac{(1-f)}{f} \boldsymbol{w}^{h}$
(B) $\quad \gamma^{h}=-\tau_{2}^{e} \frac{\epsilon \mu}{K(\epsilon) \rho_{l}} \boldsymbol{w}^{h}$
(advection term)
(C) $\quad \boldsymbol{\zeta}^{h}=-\tau_{3}^{e} \frac{\mu}{\rho \rho_{l}} \nabla \cdot\left(\nabla\left(\rho \boldsymbol{w}^{h}\right)+\left(\nabla\left(\rho \boldsymbol{w}^{h}\right)\right)^{T}\right)$
(Darcy term)
(D) $\quad \eta^{h}=\tau_{4}^{e} \nabla q^{h}$
(viscous terms)
(pressure term)

The form of the stabilizing term $\boldsymbol{\delta}^{h}$ corresponds to the classical SUPG stabilizer along with a term arising due to varying density in the mushy zone. The form of $\boldsymbol{\eta}^{h}$ corresponds to the classical PSPG stabilizer. $\zeta^{h}$ arises due to varying density in the mushy zone. The stabilizing term, $\gamma^{h}$, which we call DSPG, comes from the Darcy term and it is an important stabilizing term for the generalized Navier-Stokes/Darcy equations.

The stabilizing parameters for the advective and pressure terms are selected as follows:

$$
\begin{gathered}
\tau_{1}^{e}=\tau_{3}^{e}=\min _{\boldsymbol{x} \in \Omega^{e}}\left[\tau_{S U P G}, \frac{K(\epsilon) \rho_{l}}{\epsilon \mu}\right] \quad \text { for the convective and viscous term, } \\
\tau_{4}^{e}=\min _{\boldsymbol{x} \in \Omega^{e}}\left[\tau_{P S P G}, \frac{K(\epsilon) \rho_{l}}{\epsilon \mu}\right] \quad \text { for the pressure term }
\end{gathered}
$$

where $\tau_{S U P G}, \tau_{P S P G}$ are defined as

$$
\begin{aligned}
\tau_{S U P G} & =\frac{f h}{2\left\|\boldsymbol{v}^{h}\right\|} z(\operatorname{Re} \boldsymbol{v}) \\
\tau_{P S P G} & =\frac{f h^{\#}}{2\left\|\boldsymbol{V}^{h}\right\|} z\left(\operatorname{Re}_{\boldsymbol{V}}\right)
\end{aligned}
$$


Here, $R e \boldsymbol{v}$ and $R e \boldsymbol{V}$ are element Reynolds numbers based on the local velocity $\boldsymbol{v}^{h}$ and a global scaling velocity $\boldsymbol{V}^{h}$ as :

$$
\begin{gathered}
\operatorname{Re}_{\boldsymbol{v}}=\frac{\left\|\boldsymbol{v}^{h}\right\| h}{2 \nu} \\
\operatorname{Re}_{\boldsymbol{V}}=\frac{\left\|\boldsymbol{V}^{h}\right\| h^{\#}}{2 \nu}
\end{gathered}
$$

The expressions for element lengths $h$ and $h^{\#}$ along with those of $z(R e)$ are given in detail in references $[11,16,17,18]$ and are not repeated here. When the permeability is anisotropic, like in Equations (7) and (8), $\tau_{1}^{e}, \tau_{3}^{e}$ and $\tau_{4}^{e}$ have separate $x, y$ and $z$ components. The presence of stabilizing terms arising due to changes in density and the generalized expression taking into account isotropic or anisotropic permeability is the main difference between the current approach and the one previously described by the authors in [11]. The latter was specifically formulated for alloys where the mushy zone permeability was isotropic and whose phase densities were equal. The stabilizing parameters transition from the advective to the diffusive or Darcy dominated regime depending on the dominant underlying flow phenomenon. This makes the numerical scheme suitable for a wide class of solidification problems with strong convection.

For the Darcy term, we select $\tau_{2}^{e}$ such that the stabilization takes the form $\gamma^{h}=-(1-\epsilon) \boldsymbol{w}^{h}$, i.e.

$$
\tau_{2}^{e}=\frac{(1-\epsilon) K(\epsilon) \rho_{l}}{\epsilon \mu}
$$

Remark 1: The stabilization term $\gamma^{h}$ induced by the Darcy flow varies linearly with $\epsilon$ transitioning from $\gamma^{h}=0$ for a pure fluid phase $(\epsilon=1)$ to $\gamma^{h}=-\boldsymbol{w}^{h}$ towards a pure solid phase $(\epsilon \rightarrow 0)$. The stabilizing terms are linearized using quantities from the earlier time step or from previous iterations at the current time step. This linearization does not affect the convergence rate appreciably.

After applying the stabilized finite element method for discretizing governing equations for fluid flow, we obtain the following system of equations.

$$
\begin{gathered}
{\left[\mathbf{M}+\mathbf{M}_{\delta}+\mathbf{M}_{\gamma}+\mathbf{M}_{\zeta}\right]\{\dot{\boldsymbol{v}}\}+\left[\mathbf{N}(\boldsymbol{v})+\mathbf{N}_{\delta}(\boldsymbol{v})+\mathbf{N}_{\gamma}(\boldsymbol{v})+\mathbf{N}_{\zeta}(\boldsymbol{v})\right]\{\boldsymbol{v}\}} \\
+\left[\mathbf{K}+\mathbf{K}_{\delta}+\mathbf{K}_{\gamma}+\mathbf{K}_{\zeta}\right]\{\boldsymbol{v}\}+\left[\mathbf{D}+\mathbf{D}_{\delta}+\mathbf{D}_{\gamma}+\mathbf{D}_{\zeta}\right]\{\boldsymbol{v}\} \\
-\left[\mathbf{G}+\mathbf{G}_{\delta}+\mathbf{G}_{\gamma}+\mathbf{G}_{\zeta}\right]\{p\}=\left\{\mathbf{F}\left(T, C_{l}\right)+\mathbf{F}_{\delta}\left(T, C_{l}\right)+\mathbf{F} \gamma\left(T, C_{l}\right)+\mathbf{F}_{\zeta}\left(T, C_{l}\right)\right\}, \\
\mathbf{M}_{\eta}\{\dot{\boldsymbol{v}}\}+\left(\mathbf{M e}+\mathbf{N}_{\eta}(\boldsymbol{v})+\mathbf{K}_{\eta}+\mathbf{D}_{\eta}\right)\{\boldsymbol{v}\}+\mathbf{G}_{\eta}\{p\}=\left\{\mathbf{F}_{\eta}\left(T, C_{l}\right)+\mathbf{F}_{\eta \mathbf{1}}\right\},
\end{gathered}
$$

where $\{\boldsymbol{v}\}$ is the vector of nodal values of $\boldsymbol{v}^{h},\{\boldsymbol{\boldsymbol { v }}\}$ is the time derivative of $\{\boldsymbol{v}\}$ and $\{p\}$ is the vector of nodal values of $p^{h}$. The matrices $\mathbf{M}, \mathbf{N}(\boldsymbol{v}), \mathbf{K}, \mathbf{D}$ and $\mathbf{G}$ are derived from the time-dependent, advective, viscous, Darcy and pressure terms, respectively. All these matrices incorporate changes arising due to varying density in the mushy zone. $\mathbf{F}_{\eta \mathbf{1}}$ in Equation (48) represents the forcing quantity due to rate of change of density $\left(\rho^{n}-\rho^{n-1}\right) / \Delta t$. Subscripts $\delta$, $\eta, \gamma$ and $\zeta$ identify the SUPG, PSPG, DSPG and viscous stabilizing terms, respectively. The subscript $p$ is used here to denote the global velocity degree of freedom in the final system of equations that corresponds to the $i^{\text {th }}$ velocity degree of freedom of the elemental node $\alpha$ $\left(\alpha=1, \ldots, n_{\text {nodes }}^{e}\right)$ in the $e^{t h}$ element. Similarly, one can define $q$. We also introduce $r$ and $s$ 
to denote the global pressure degrees of freedom. We denote the SUPG contribution as $P_{\alpha}^{e}$, the PSPG contribution in the $i^{t h} \quad\left(i=1, \ldots, n_{s d}\right)$ direction as $E_{\alpha i}^{e}$, DSPG contribution as $D_{\alpha}^{e}$. They are defined as follows:

$$
\begin{aligned}
P_{\alpha}^{e} & =\frac{1}{f} \tau_{1}^{e} \boldsymbol{v}^{h} \cdot \nabla N_{\alpha}^{e} \\
E_{\alpha i}^{e} & =\tau_{4}^{e} N_{\alpha, i}^{e} \\
D_{\alpha}^{e} & =-(1-\epsilon) N_{\alpha}^{e}
\end{aligned}
$$

$Z_{\alpha}^{e}$ denotes the contribution from viscous terms. After simplifying and neglecting some terms, it takes the following form:

$$
Z_{\alpha}^{e}=\tau_{1}^{e} \frac{2 \mu}{\rho \rho_{l}} \nabla \rho \cdot \nabla N_{\alpha}^{e}
$$

Matrices and vectors in Equations (47) and (48) are described below.

$$
\begin{aligned}
& {\left[\mathbf{M}+\mathbf{M}_{\delta}+\mathbf{M}_{\gamma}+\mathbf{M}_{\zeta}\right]_{p q}=\underset{e=1}{n_{e l}} \int_{\Omega^{e}} \rho\left(N_{\alpha}^{e}+P_{\alpha}^{e}+D_{\alpha}^{e}+Z_{\alpha}^{e}\right) N_{\beta}^{e} \delta_{i j} d \Omega^{e}} \\
& {\left[\mathbf{N}(\boldsymbol{v})+\mathbf{N}_{\delta}(\boldsymbol{v})+\mathbf{N}_{\gamma}(\boldsymbol{v})+\mathbf{N}_{\zeta}(\boldsymbol{v})\right]_{p q}=\underset{e=1}{n_{e l}} \int_{\Omega^{e}} \frac{\rho}{f}\left(N_{\alpha}^{e}+P_{\alpha}^{e}+D_{\alpha}^{e}+Z_{\alpha}^{e}\right) N_{\beta, k}^{e} v_{k} \delta_{i j} d \Omega^{e}} \\
& -{\stackrel{\mathbf{A}}{n_{e l}}}_{e=1} \int_{\Omega^{e}} \frac{\partial \rho}{\partial t} \frac{(1-f)}{f}\left(N_{\alpha}^{e}+P_{\alpha}^{e}+D_{\alpha}^{e}+Z_{\alpha}^{e}\right) N_{\beta}^{e} \delta_{i j} d \Omega^{e} \\
& {\left[\mathbf{K}+\mathbf{K}_{\delta}+\mathbf{K}_{\gamma}+\mathbf{K}_{\zeta}\right]_{p q}=\underset{e=1}{n_{e l}} \int_{\Omega^{e}} \frac{\mu \rho}{\rho_{l}}\left(N_{\alpha, k}^{e} N_{\beta, k}^{e} \delta_{i j}+N_{\alpha, j}^{e} N_{\beta, i}^{e}\right) d \Omega^{e}} \\
& +\mathbf{A}_{e=1}^{n_{e l}} \int_{\Omega^{e}} \frac{\mu}{\rho_{l}}\left(\rho_{, k} N_{\alpha, k}^{e} \delta_{i j}+N_{\alpha, j}^{e} \rho_{, i}\right) N_{\beta}^{e} d \Omega^{e} \\
& -\quad{\stackrel{\mathbf{A}}{n_{e l}}}_{e=1} \int_{\Omega^{e}} \frac{\mu}{\rho_{l}}\left(P_{\alpha}^{e}+D_{\alpha}^{e}+Z_{\alpha}^{e}\right)\left(2 N_{\beta, k}^{e} \rho_{, k} \delta_{i j}+N_{\beta, i}^{e} \rho_{, j}+N_{\beta, j}^{e} \rho_{, i}\right) d \Omega^{e} \\
& {\left[\mathbf{D}+\mathbf{D}_{\delta}+\mathbf{D}_{\gamma}+\mathbf{D}_{\zeta}\right]_{p q}=\underset{e=1}{n_{e l}} \int_{\Omega^{e}} \frac{\epsilon \mu \rho}{K(\epsilon) \rho_{l}}\left(N_{\alpha}^{e}+P_{\alpha}^{e}+D_{\alpha}^{e}+Z_{\alpha}^{e}\right) N_{\beta}^{e} \delta_{i j} d \Omega^{e}} \\
& {\left[\mathbf{G}+\mathbf{G}_{\delta}+\mathbf{G}_{\gamma}+\mathbf{G}_{\zeta}\right]_{p s}=\underset{e=1}{n_{e l}} \int_{\Omega^{e}}\left(N_{\alpha, i}^{e} N_{\beta}^{e}-\left(P_{\alpha}^{e}+D_{\alpha}^{e}+Z_{\alpha}^{e}\right) N_{\beta, i}^{e}\right) d \Omega^{e}} \\
& {\left[\mathbf{F}+\mathbf{F}_{\delta}+\mathbf{F}_{\gamma}+\mathbf{F}_{\zeta}\right]_{p}=\stackrel{n_{e l}}{\mathbf{A}} \int_{\Omega^{e}}-\epsilon \rho_{l_{0}} g\left(N_{\alpha}^{e}+P_{\alpha}^{e}+D_{\alpha}^{e}+Z_{\alpha}^{e}\right)\left(\beta_{T}\left(T^{e}-T_{0}\right)+\beta_{s}\left(C_{l}^{e}-C_{l_{0}}\right)\right) \boldsymbol{e}_{\boldsymbol{g}_{i}} d \Omega^{e}} \\
& {[\mathbf{M e}]_{r q}=\underset{e=1}{n_{e l}} \int_{\Omega^{e}} N_{\alpha}^{e}\left(\rho N_{\beta, j}^{e}+\rho_{, j} N_{\beta}\right) d \Omega^{e}} \\
& {\left[\mathbf{M}_{\eta}\right]_{r q}=\underset{e=1}{n_{e l}} \int_{\Omega^{e}} \rho E_{\alpha j}^{e} N_{\beta}^{e} d \Omega^{e}}
\end{aligned}
$$




$$
\begin{aligned}
& {\left[\mathbf{N}_{\eta}(\boldsymbol{v})\right]_{r q}=\underset{e=1}{n_{e l}} \int_{\Omega^{e}}\left(\frac{\rho}{f} E_{\alpha j}^{e} v_{k} N_{\beta, k}^{e}-\frac{1-f}{f} \frac{\partial \rho}{\partial t} E_{\alpha j}^{e} N_{\beta}^{e}\right) d \Omega^{e}} \\
& {\left[\mathbf{K}_{\eta}\right]_{r q}=\underset{e=1}{n_{e l}} \int_{\Omega^{e}}-\frac{\mu}{\rho_{l}}\left(2 N_{\beta, k}^{e} \rho_{, k} E_{\alpha j}^{e}+N_{\beta, k}^{e} E_{\alpha k}^{e} \rho_{, j}+E_{\alpha k}^{e} \rho_{, k} N_{\beta, j}^{e}\right) d \Omega^{e}} \\
& {\left[\mathbf{D}_{\eta}\right]_{r q}=\underset{e=1}{n_{e l}} \int_{\Omega^{e}} \frac{\epsilon \mu \rho}{K(\epsilon) \rho_{l}} E_{\alpha j}^{e} N_{\beta}^{e} d \Omega^{e}} \\
& {\left[\mathbf{G}_{\eta}\right]_{r s}=\underset{e=1}{n_{e l}} \int_{\Omega^{e}} E_{\alpha k}^{e} N_{\beta, k}^{e} d \Omega^{e}} \\
& {\left[\mathbf{F}_{\eta}\right]_{r}=\underset{e=1}{n_{e l}} \int_{\Omega^{e}}-\epsilon \rho_{l_{0}} g E_{\alpha k}^{e} \boldsymbol{e}_{\boldsymbol{g}_{k}}\left(\beta_{T}\left(T^{e}-T_{0}\right)+\beta_{s}\left(C_{l}^{e}-C_{l_{0}}\right)\right) d \Omega^{e}} \\
& {\left[\mathbf{F}_{\eta \mathbf{1}}\right]_{r}=-\underset{e=1}{n_{e l}} \int_{\Omega^{e}} N_{\alpha}^{e} \frac{\partial \rho}{\partial t} d \Omega^{e}}
\end{aligned}
$$

where $\delta_{i j}$ stands for the Kronecker delta and $\frac{\partial \rho}{\partial t}$ is expressed as $\left(\rho^{n}-\rho^{n-1}\right) / \Delta t$. Terms involving second derivatives of shape functions have been eliminated because of our use of bilinear elements. The time integration of Equations $(47,48)$ is performed using a backward Euler scheme with $\boldsymbol{v}$ expressed as

$$
\dot{v}=\frac{\boldsymbol{v}^{n}-\boldsymbol{v}^{n-1}}{\Delta t}
$$

The volume-averaged pressure gradient term $-\epsilon \nabla\left\langle p_{l}\right\rangle^{l}$, where $\left\langle p_{l}\right\rangle^{l}$ denotes the intrinsic liquid pressure, is expressed in Equation (2) using $\epsilon\left\langle p_{l}\right\rangle^{l}=p$. This modification, previously mentioned in [11], eliminates $\epsilon$ from the diagonal term $G_{\eta}$ in Equation (64) and helps in improving the convergence rate of the fluid flow solver. The solution procedure, which is similar to that described in [11], is not repeated here.

\section{FINITE ELEMENT SCHEME FOR THE ENERGY AND SPECIES EQUATIONS}

A classical SUPG formulation is used for the solution of the energy and species conservation equations. The energy and species equations are first cast into the standard form of a convection-diffusion scalar equation. We first consider the energy equation where the enthalpy $h$ is the main unknown to be solved. Using Equations (9)-(12), we get the following expression relating $h_{l}$ and $h$ :

$$
h_{l}-h=(1-f)\left[\left(c_{l}-c_{s}\right)\left(T-T_{e u t}\right)+h_{f}\right]
$$

We can approximate $\nabla T$ as follows:

$$
\nabla T^{n} \approx \alpha^{n-1} \nabla h^{n}
$$

with $n$ denoting the time step and $\alpha^{n}$ is defined element-wise as:

$$
\alpha^{n}=\left\{\begin{array}{cc}
\frac{\nabla T^{n}(\boldsymbol{x}) \cdot \nabla h^{n}(\boldsymbol{x})}{\left|\nabla h^{n}(\boldsymbol{x})\right|^{2}} & \text { if }\left|\nabla h^{n}(\boldsymbol{x})\right|>0, \\
\frac{d T}{d h}(\boldsymbol{x}) & \text { if }\left|\nabla h^{n}(\boldsymbol{x})\right|=0,
\end{array}\right.
$$

Copyright (c) 2005 John Wiley \& Sons, Ltd.

Int. J. Numer. Meth. Engng 2005; :1-28 
Using Equations (1), (68), and the above approximation, Equation (3) is written as:

$$
\begin{aligned}
\left.\rho \frac{\partial h(\boldsymbol{x}, t)}{\partial t}+\rho \boldsymbol{v}(\boldsymbol{x}, t) \cdot \nabla h(\boldsymbol{x}, t)\right) & =-\nabla \cdot\left[\rho \boldsymbol{v}(\boldsymbol{x}, t)(1-f)\left(\left(c_{l}-c_{s}\right)\left(T(\boldsymbol{x}, t)-T_{\text {eut }}\right)+h_{f}\right)\right] \\
+\nabla \cdot\left[\left(\epsilon k_{l}\right.\right. & \left.\left.+(1-\epsilon) k_{s}\right) \alpha \nabla h(\boldsymbol{x}, t)\right]
\end{aligned}
$$

We also define the following finite element interpolation function space for $h^{h}$ :

$$
S_{H}^{h}=\left\{h^{h}\left|h^{h} \in C^{o}(\bar{\Omega}), h^{h}\right|_{\Omega^{e}} \in P^{l}\left(\Omega^{e}\right), e=1,2, \ldots, n_{e l}, h^{h} \doteq 0 \text { on } \Gamma\right\}
$$

The weak formulation of Equation (70) can be stated as follows: Find $h^{h} \in S_{H}^{h}$ such that, $\forall \hat{w}^{h} \in S_{H}^{h}$ :

$$
\begin{array}{r}
\int_{\Omega} \hat{w}^{h}\left(\rho \frac{\partial h^{h}}{\partial t}+\rho \boldsymbol{v}^{h} \cdot \nabla h^{h}\right) d \Omega \\
+\int_{\Omega} \nabla \hat{w}^{h} \cdot\left(\left(\epsilon k_{l}+(1-\epsilon) k_{s}\right) \alpha \nabla h^{h}\right) d \Omega \\
+\underset{e=1}{n_{e l}} \int_{\Omega^{e}} \hat{\delta}^{h}\left(\rho \frac{\partial h^{h}}{\partial t}+\rho \boldsymbol{v}^{h} \cdot \nabla h^{h}\right) d \Omega^{e} \\
=-\int_{\Omega} \hat{w}^{h} \nabla \cdot\left[\rho \boldsymbol{v}^{h}(1-f)\left(\left(c_{l}-c_{s}\right)\left(T^{h}-T_{\text {eut }}\right)+h_{f}^{h}\right)\right] d \Omega \\
-\underset{e=1}{n_{e l}} \int_{\Omega^{e}} \hat{\delta}^{h} \nabla \cdot\left[\rho \boldsymbol{v}^{h}(1-f)\left(\left(c_{l}-c_{s}\right)\left(T^{h}-T_{\text {eut }}\right)+h_{f}^{h}\right)\right] d \Omega^{e}
\end{array}
$$

The spatial discretization of Equation (72) leads to the following ordinary differential equation:

$$
\left[\hat{\mathbf{M}}+\hat{\mathbf{M}}_{\delta}\right]\{\dot{h}\}+\left[\hat{\mathbf{N}}+\hat{\mathbf{N}}_{\delta}\right]\{h\}+\left[\hat{\mathbf{K}}+\hat{\mathbf{K}}_{\delta}\right]\{h\}=\left\{\hat{\mathbf{F}}+\hat{\mathbf{F}}_{\delta}\right\}
$$

The element stiffness matrices and the load vector are given as follows:

$$
\begin{aligned}
& {\left[\hat{\mathbf{M}}+\hat{\mathbf{M}}_{\delta}\right]_{p q}=\underset{e=1}{n_{e l}} \int_{\Omega^{e}} \rho\left(N_{\alpha}^{e}+\delta_{\alpha}^{e}\right) N_{\beta}^{e} d \Omega^{e}} \\
& {\left[\hat{\mathbf{N}}(\boldsymbol{v})+\hat{\mathbf{N}}_{\delta}(\boldsymbol{v})\right]_{p q}=\underset{e=1}{n_{e l}} \int_{\Omega^{e}} \rho\left(N_{\alpha}^{e}+\delta_{\alpha}^{e}\right) v_{k}^{e} N_{\beta, k}^{e} d \Omega^{e}} \\
& {\left[\hat{\mathbf{K}}+\hat{\mathbf{K}}_{\delta}\right]_{p q}=\underset{e=1}{n_{e l}} \int_{\Omega^{e}}\left(\epsilon k_{l}+(1-\epsilon) k_{s}\right) \alpha N_{\alpha, k}^{e} N_{\beta, k}^{e} d \Omega^{e}}
\end{aligned}
$$

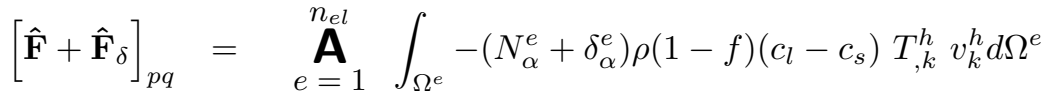

$$
\begin{aligned}
& +\int_{\Omega^{e}}\left(N_{\alpha}^{e}+\delta_{\alpha}^{e}\right) \rho v_{k}^{h} f_{, k}\left(\left(c_{l}-c_{s}\right)\left(T^{h}-T_{e u t}\right)+h_{f}\right) d \Omega^{e} \\
& +\int_{\Omega^{e}}\left(N_{\alpha}^{e}+\delta_{\alpha}^{e}\right) \frac{\partial \rho}{\partial t}(1-f)\left(\left(c_{l}-c_{s}\right)\left(T^{h}-T_{e u t}\right)+h_{f}\right) d \Omega^{e}
\end{aligned}
$$

Using a similar argument, we rewrite Equation (4) as:

$$
\begin{aligned}
\rho \frac{\partial C(\boldsymbol{x}, t)}{\partial t}+\left(C(\boldsymbol{x}, t)-C_{l}(\boldsymbol{x}, t)\right) \frac{\partial \rho}{\partial t} & +\rho \boldsymbol{v}(\boldsymbol{x}, t) \cdot \nabla C_{l}(\boldsymbol{x}, t)=\nabla \cdot\left(D^{*} \rho \nabla C(\boldsymbol{x}, t)\right) \\
& +\nabla \cdot\left(\rho D^{*} \nabla\left(C_{l}(\boldsymbol{x}, t)-C(\boldsymbol{x}, t)\right)\right)
\end{aligned}
$$


where $D^{*}=f D_{l}$. Note that, like its energy and momentum counterparts, this equation too has terms arising due to changes in density.

The weak formulation of Equation (78) can be stated as follows: Find $C^{h} \in S_{C}^{h}$ such that, $\forall \tilde{w}^{h} \in S_{C}^{h}$ :

$$
\begin{gathered}
\int_{\Omega} \tilde{w}^{h}\left(\rho \frac{\partial C^{h}}{\partial t}+\rho \boldsymbol{v}^{h} \cdot \nabla C_{l}^{h}+\frac{\partial \rho}{\partial t}\left(C^{h}-C_{l}^{h}\right)\right) d \Omega+\int_{\Omega} \nabla \tilde{w}^{h} \cdot\left(\rho D^{*} \nabla C^{h}\right) d \Omega \\
+\underset{e=1}{n_{e l}} \int_{\Omega^{e}} \tilde{\delta}^{h}\left(\rho \frac{\partial C^{h}}{\partial t}+\rho \boldsymbol{v}^{h} \cdot \nabla C_{l}^{h}+\frac{\partial \rho}{\partial t}\left(C^{h}-C_{l}^{h}\right)\right) d \Omega^{e} \\
=\int_{\Omega} \tilde{w}^{h} \nabla \cdot\left(\rho D^{*} \nabla\left(C_{l}^{h}-C^{h}\right)\right) d \Omega+\underset{e=1}{n_{e l}} \int_{\Omega^{e}} \tilde{\delta}^{h} \nabla \cdot\left(\rho D^{*} \nabla\left(C_{l}^{h}-C^{h}\right)\right) d \Omega^{e}
\end{gathered}
$$

The spatial discretization of Equation (79) leads to the following ordinary differential equation:

$$
\left[\tilde{\mathbf{M}}+\tilde{\mathbf{M}}_{\delta}\right]\{\dot{C}\}+\left[\tilde{\mathbf{M}}_{\mathbf{1}}+\tilde{\mathbf{M}}_{\mathbf{1} \delta}\right]\{C\}+\left[\tilde{\mathbf{N}}+\tilde{\mathbf{N}}_{\delta}\right]\left\{C_{l}\right\}+\left[\tilde{\mathbf{K}}+\tilde{\mathbf{K}}_{\delta}\right]\{C\}=\left\{\tilde{\mathbf{F}}+\tilde{\mathbf{F}}_{\delta}\right\}
$$

The element stiffness matrices and the load vector are given as follows:

$$
\begin{aligned}
{\left[\tilde{\mathbf{M}}+\tilde{\mathbf{M}}_{\delta}\right]_{p q} } & =\underset{e=1}{n_{e l}} \int_{\Omega^{e}} \rho\left(N_{\alpha}^{e}+\delta_{\alpha}^{e}\right) N_{\beta}^{e} d \Omega^{e} \\
{\left[\tilde{\mathbf{M}}_{\mathbf{1}}+\tilde{\mathbf{M}}_{\mathbf{1} \delta}\right]_{p q} } & =\underset{e=1}{n_{e l}} \int_{\Omega^{e}} \frac{\partial \rho}{\partial t}\left(N_{\alpha}^{e}+\delta_{\alpha}^{e}\right) N_{\beta}^{e} d \Omega^{e} \\
{\left[\tilde{\mathbf{N}}(\boldsymbol{v})+\tilde{\mathbf{N}}_{\delta}(\boldsymbol{v})\right]_{p q} } & =\underset{e=1}{n_{e l}} \int_{\Omega^{e}} \rho\left(N_{\alpha}^{e}+\delta_{\alpha}^{e}\right) v_{k}^{e} N_{\beta, k}^{e} d \Omega^{e} \\
{\left[\tilde{\mathbf{K}}+\tilde{\mathbf{K}}_{\delta}\right]_{p q} } & =\underset{e=1}{n_{e l}} \int_{\Omega^{e}} \rho D^{*} N_{\alpha, k}^{e} N_{\beta, k}^{e} d \Omega^{e} \\
{\left[\tilde{\mathbf{F}}+\tilde{\mathbf{F}}_{\delta}\right]_{p q} } & =\underset{e=1}{n_{e l}} \int_{\Omega^{e}}\left(N_{\alpha}^{e}+\delta_{\alpha}^{e}\right) C_{l} \frac{\partial \rho}{\partial t} d \Omega^{e}
\end{aligned}
$$

Let us denote:

$$
\hat{\mathbf{M}}=\left[\tilde{\mathbf{M}}+\tilde{\mathbf{M}}_{\delta}\right]
$$

with similar notation being used for $\hat{\mathbf{N}}, \hat{\mathbf{K}}$, and $\hat{\mathbf{F}}$. The resulting non-linear equations for enthalpy and solute concentration can be expressed as:

$$
\begin{aligned}
\hat{\mathbf{M}}\left\{\dot{h}_{n}\right\} & +\left(\hat{\mathbf{N}}\left(\boldsymbol{v}_{n}\right)+\hat{\mathbf{K}}\right)\left\{h_{n}\right\}=\hat{\mathbf{F}}_{\mathbf{T}} \\
\hat{\mathbf{M}}\left\{\dot{C}_{n}\right\} & +\left(\tilde{\mathbf{M}}_{\mathbf{1}}+\hat{\mathbf{K}}\right)\left\{C_{n}\right\}+\hat{\mathbf{N}}\left(\boldsymbol{v}_{n}\right)\left\{C_{l n}\right\}=\hat{\mathbf{F}}_{\mathbf{C}}
\end{aligned}
$$

Incorporating the viscous term, $\hat{\mathbf{K}}$, into the mass matrix of the predictor-corrector algorithm $\left(\mathbf{M}=\hat{\mathbf{M}}+\gamma \Delta t\left(\tilde{\mathbf{M}}_{\mathbf{1}}+\hat{\mathbf{K}}\right)\right)$ for Equation (88), with $\Delta \dot{C}$ as the main unknown, helps in improving the convergence rate of the solute sub-problem. 


\subsection{Calculation of shrinkage}

When densities of the solid and liquid phases are different, decrease in volume or shrinkage occurs due to progressive phase change. To feed the resulting volume contraction, a riser is provided near the insulated end. Our approach here is different from that of [10], where an inlet velocity boundary condition was provided on a portion of the top surface. Feeding velocity was calculated from the volume required to compensate shrinkage. In our work, liquid from the riser feeds the shrinkage and this leads to an effective reduction in the domain size. The change in volume at each time step is calculated according to the following relation, previously described in [8] :

$$
\Delta_{v}=\int_{\Omega} \frac{\rho_{s}-\rho_{l}}{\rho_{l}} \frac{\partial \epsilon}{\partial t} d \Omega
$$

To account for this change, the top surface representing the riser is moved downwards like a rigid lid whose velocity is determined from $\Delta_{v}$. This approach neglects any changes in curvature that may occur at the top surface due to surface tension effects.

\section{COUPLING OF THE VARIOUS SUB-PROBLEMS AND OVERALL TIME INTEGRATION}

Various sub-problems considered here include the thermal, flow, and solute sub-problems as well as update formulae based on thermodynamic and two-phase relationships. The error criterion is based on the relative error in the solutions obtained between iterations within a time step. For example, in the fluid flow solver, the error norm is defined as $\left|\Delta U^{i+1}\right| /\left|U_{n}^{i+1}\right|$ where $U=[\boldsymbol{v}, p]$. The overall time integration process summarized below:

1. At time $t_{n}$, all fields such as velocity $\boldsymbol{v}_{n}$, enthalpy $h_{n}$, temperature $T_{n}$, concentration $C_{n}$, liquid mass and volume fractions $f_{n}$ and $\epsilon_{n}$, density $\rho_{n}$ etc. are known.

2. Advance to time step $t_{n+1}=t_{n}+\Delta t$. Set $\mathrm{j}=0, \boldsymbol{v}_{n+1}^{j=0}=\boldsymbol{v}_{n}, h_{n+1}^{j=0}=h_{n}, f_{n+1}^{j=0}=f_{n}$, $C_{n+1}^{j=0}=C_{n}$ etc., where $j$ is an iteration index and subscripts $n, n+1$ denote time levels.

3. Compute the mixture enthalpy $h_{n+1}^{j+1}$ using the algorithm described in Section 4 .

4. Compute the mixture concentration $C_{n+1}^{j+1}$ using the algorithm described in Section 4.

5. Update temperature $T_{n+1}^{j+1}$, liquid concentration $C_{l_{n+1}^{j+1}}$, liquid mass and volume fractions $f_{n+1}^{j+1}$ and $\epsilon_{n+1}^{j+1}$ and density $\rho_{n+1}^{j+1}$.

6. Repeat steps 3 to 5 so that $\left\|T_{n+1}^{j+1}-T_{n+1}^{j}\right\|<\epsilon_{\text {tol }},\left\|C_{l n+1}^{j+1}-C_{l n+1}^{j}\right\|<\epsilon_{t o l},\left\|f_{n+1}^{j+1}-f_{n+1}^{j}\right\|<$ $\epsilon_{t o l}$ are all satisfied $\left(\epsilon_{t o l}=10^{-8}\right.$ here denotes appropriate tolerance). If convergence is satisfied, set $h_{n+1}=h_{n+1}^{j+1}, C_{n+1}=C_{n+1}^{j+1}$ and $f_{n+1}=f_{n+1}^{j+1}$.

7. Compute the mixture velocity $\boldsymbol{v}_{n+1}^{j+1}$ and pressure $p_{n+1}^{j+1}$ using the algorithm of Section 3 . Upon convergence, set $\boldsymbol{v}_{n+1}=\boldsymbol{v}_{n+1}^{j+1}, n=n+1$ and proceed to step 2 .

The momentum equations are decoupled and solved only once in the overall time integration loop. The energy and solute equations are solved multiple times in an inner iteration loop. Both of these processes help in increasing the overall convergence rates of the individual solvers. For the solution of the energy and solute sub-problems, a multi-step predictor-corrector method is used. For the solution of the momentum sub-problem, the Newton-Raphson method is used. A parallel matrix free solver based on the GMRES algorithm developed in [19], is employed 


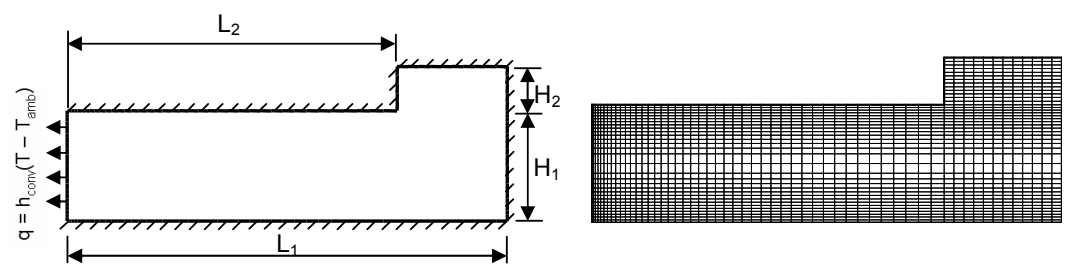

(a)

(b)

Figure 2. (a) Domain and (b) finite element mesh for Example 1.

for all our numerical examples. By eliminating the need to assemble and LU factorize global matrices, significant speed up is obtained besides reduction in memory requirements. In the initial time steps, convergence is typically obtained in 3 to 4 iterations. Later in the solution process, convergence is obtained in 2 iterations for all sub-problems. All problems considered were run on a parallel Windows-based cluster in the Cornell Theory Center (CTC) consisting of Intel Pentium IV $2.4 \mathrm{GHz}$ processors. The number of processors used for Examples 1 and 2 (two-dimensional problems) was 10 and 16, respectively, whereas 30 processors were used for the three-dimensional problem (Example 3).

\section{NUMERICAL EXAMPLES}

Three examples are considered in this section including a three-dimensional vertical solidification example. Finite element meshes for all problems considered are made using bilinear quadrilateral elements.

\subsection{Example 1: Horizontal solidification of Lead-Tin alloy}

With the aim of testing and validating our mathematical model and numerical algorithm, a binary alloy solidification problem is solved where densities of solid and liquid phases are different. The domain is shown in Fig. 2. $\mathrm{L}_{1}, \mathrm{~L}_{2}, \mathrm{H}_{1}$ and $\mathrm{H}_{2}$ are taken as $0.2 \mathrm{~m}, 0.15 \mathrm{~m}, 0.05$ $\mathrm{m}$ and $0.02 \mathrm{~m}$, respectively. The left wall is subjected to convective cooling condition with the heat transfer coefficient, $h_{\text {conv }}$, being $1000 \mathrm{Wm}^{-2 o} \mathrm{C}^{-1}$. No slip boundary conditions are applied on all walls and the melt is initially assumed to be stationary and at a constant initial temperature, $T_{i}$.

We consider the solidification of an alloy whose composition is $19.2 \%$ Tin with the rest Lead. This problem was addressed earlier in [10] using finite difference methods. The authors in [10] used an inlet flow condition instead of a riser to compensate the volume change arising due to shrinkage. Important physical constants for this alloy are summarized in Table I. The alloy considered is characterized by a wide freezing range of approximately $100{ }^{\circ} \mathrm{C}$. Tin, which is lighter than Lead, is rejected from the solid phase with progressive solidification. Consequently, thermal and solutal convection oppose each other. Moreover, due to different densities of the solid and liquid phases, shrinkage driven flow is present in the mushy zone. This problem is implemented with two meshes consisting of 1150 and 1800 elements to study convergence. Comparison between maximum velocities and midplane $\left(y=\mathrm{H}_{1} / 2\right)$ solute concentrations are 
Table I. Important physical parameters for the solidification of Lead-Tin alloy (taken from [10]).

\begin{tabular}{|c|c|c|c|}
\hline Physical parameter & Symbol & Value & Units \\
\hline Heat conductivity in solid & $k_{s}$ & $3.97 \times 10^{-2}$ & $k W m^{-1 o} C^{-1}$ \\
\hline Heat conductivity in melt & $k_{l}$ & $2.29 \times 10^{-2}$ & $k W m^{-1 o} C^{-1}$ \\
\hline Specific heat in solid & $c_{s}$ & 0.1779 & $k J k g^{-1 o} C^{-1}$ \\
\hline Specific heat in melt & $c_{l}$ & 0.1547 & $k J k g^{-1 o} C^{-1}$ \\
\hline Latent heat & $h_{f}$ & 30.162 & $k J k g^{-1}$ \\
\hline Partition coefficient & $\kappa_{p}$ & 0.31 & \\
\hline Thermal expansion coefficient & $\beta_{T}$ & $1.09 \times 10^{-4}$ & ${ }^{o} C^{-1}$ \\
\hline Solutal expansion coefficient & $\beta_{s}$ & 0.354 & ${ }^{o} C^{-1}$ \\
\hline Density of solid & $\rho_{s}$ & 10800 & $k g m^{-3}$ \\
\hline Density of liquid & $\rho_{l}$ & 10000 & $k g \mathrm{~m}^{-3}$ \\
\hline Viscosity & $\mu$ & 0.0023 & $k g m^{-1} s^{-1}$ \\
\hline Eutectic temperature & $T_{\text {eut }}$ & 183 & ${ }^{o} \mathrm{C}$ \\
\hline Melting temperature & $T_{m}$ & 327 & ${ }^{\circ} \mathrm{C}$ \\
\hline Initial temperature & $T_{i}$ & 287 & ${ }^{o} \mathrm{C}$ \\
\hline Ambient temperature & $T_{a m b}$ & 20 & ${ }^{o} \mathrm{C}$ \\
\hline Initial concentration & $C_{l, 0}$ & 19.2 & $w t \%$ \\
\hline Gravity & $\mathrm{g}$ & 9.81 & $m s^{-2}$ \\
\hline Slope of liquidus line & $m_{l i q}$ & -232.63 & ${ }^{o} \mathrm{C}$ \\
\hline Liquid diffusion coefficient & $D_{l}$ & $1.05 \times 10^{-9}$ & $m^{2} s^{-1}$ \\
\hline
\end{tabular}

Table II. Comparison of magnitudes of maximum velocities for both grids in Example 1.

\begin{tabular}{lcr}
\hline Simulation time & $\begin{array}{c}|v|_{\max }(\text { grid 1) } \\
\mathrm{mm} / \mathrm{s}\end{array}$ & $|v|_{\max }($ grid 2) \\
$\mathrm{mm} / \mathrm{s}$
\end{tabular}

given in Tables II and III, respectively, for both grids at different times. A fairly good match is obtained. The same problem is repeated after neglecting shrinkage driven flow by assuming densities of both the phases to be equal. Physical parameters are assumed to be the same for both cases.

Figure 3 shows mid-height concentration profiles at different times. When compared with corresponding plots in [10] for buoyancy driven flow and combined buoyancy and shrinkage flows, a fairly good match is obtained with small quantitative differences. This is also summarized in Table IV where maximum and minimum midplane concentrations of Tin are compared with those plotted in [10] at different times for the combined flow case. Figures 4-7 show solute distribution, liquid solute concentration, liquid mass fraction and temperature at different times. Solute enrichment occurs near the top surface of the cavity and solute depletion near the bottom surface. Effects of shrinkage driven flow are prominent in regions where the 
Table III. Comparison of maximum and minimum midplane concentrations for both grids in Example

\begin{tabular}{lccrrr}
\hline & \multicolumn{2}{c}{1.} & & \multicolumn{2}{c}{$C_{\text {grid } 2}$} \\
Simulation time & $C_{\max }$ & $C_{\min }$ & $C_{\max }$ & $C_{\min }$ \\
\hline 25.0 & 0.1972 & 0.1902 & 0.1970 & 0.1901 \\
50.0 & 0.2005 & 0.1884 & 0.1998 & 0.1884 \\
100.0 & 0.2011 & 0.1867 & 0.2014 & 0.1864 \\
200.0 & 0.2019 & 0.1834 & 0.2017 & 0.1832 \\
400.0 & 0.2017 & 0.1744 & 0.2016 & 0.1741 \\
600.0 & 0.2019 & 0.1736 & 0.2023 & 0.1713 \\
\hline
\end{tabular}

Table IV. Comparison between maximum and minimum midplane concentration of Tin calculated here with grid 2 and those reported in [10] for a similar example.

\begin{tabular}{lccccc}
\hline & \multicolumn{2}{c}{$C_{\text {calculated }}$} & \multicolumn{2}{c}{$C_{\text {reported }}$} \\
Simulation time & $C_{\max }$ & $C_{\min }$ & $C_{\max }$ & $C_{\min }$ \\
\hline 25.0 & 0.197 & 0.190 & 0.198 & 0.190 \\
50.0 & 0.200 & 0.188 & 0.200 & 0.188 \\
100.0 & 0.201 & 0.186 & 0.201 & 0.184 \\
200.0 & 0.202 & 0.183 & 0.202 & 0.180 \\
400.0 & 0.202 & 0.174 & 0.202 & 0.167 \\
\hline
\end{tabular}

temperature approaches the solidus temperature. This is evident from midplane segregation plots in Fig. 3, where there is sharp rise in concentration of Tin near the left wall. However, when shrinkage driven flows are neglected, there is no such rise in the midplane concentrations as observed in Fig. 3 for only buoyancy driven flows. Thermal and solutal buoyancy driven flows dominate in other regions. As the mushy zone slowly develops, buoyancy driven flows expel Tin-rich liquid into the melt thereby producing a large zone of negative segregation in the mush. In Figs. 3e and 3f discontinuities, similar to those shown in [10], are observed. These are attributed to the localized remelting that occurs as solidification progresses and contribute to further segregation. Effects of convective activity and severe segregation are also observed in isotherms and liquid concentration profiles. Both of them are significantly distorted near the top and bottom surfaces. The overall CPU time for examples in this section was around 78 hours.

\subsection{Example 2: Vertical two-dimensional solidification of a Lead-Tin alloy}

Vertical solidification of a Lead-Tin alloy is considered next where the mushy zone is characterized by an anisotropic permeability. Equations (7) and (8) describe permeability of the mushy zone in directions transverse and parallel to dendrite growth, respectively. To maintain consistency between the numerical model discussed here and that used in the previous examples, we use the same segregation model viz. lever rule for examples in this section too. The alloy being solidified has a composition 23.4\% Tin with the rest Lead. The two-dimensional problem domain along with boundary conditions is shown in Fig. 8. The cavity is $0.007 \mathrm{~m}$ wide and $0.04 \mathrm{~m}$ high. Initially, the bottom surface is at $272.5{ }^{\circ} \mathrm{C}$. An initial vertical thermal 

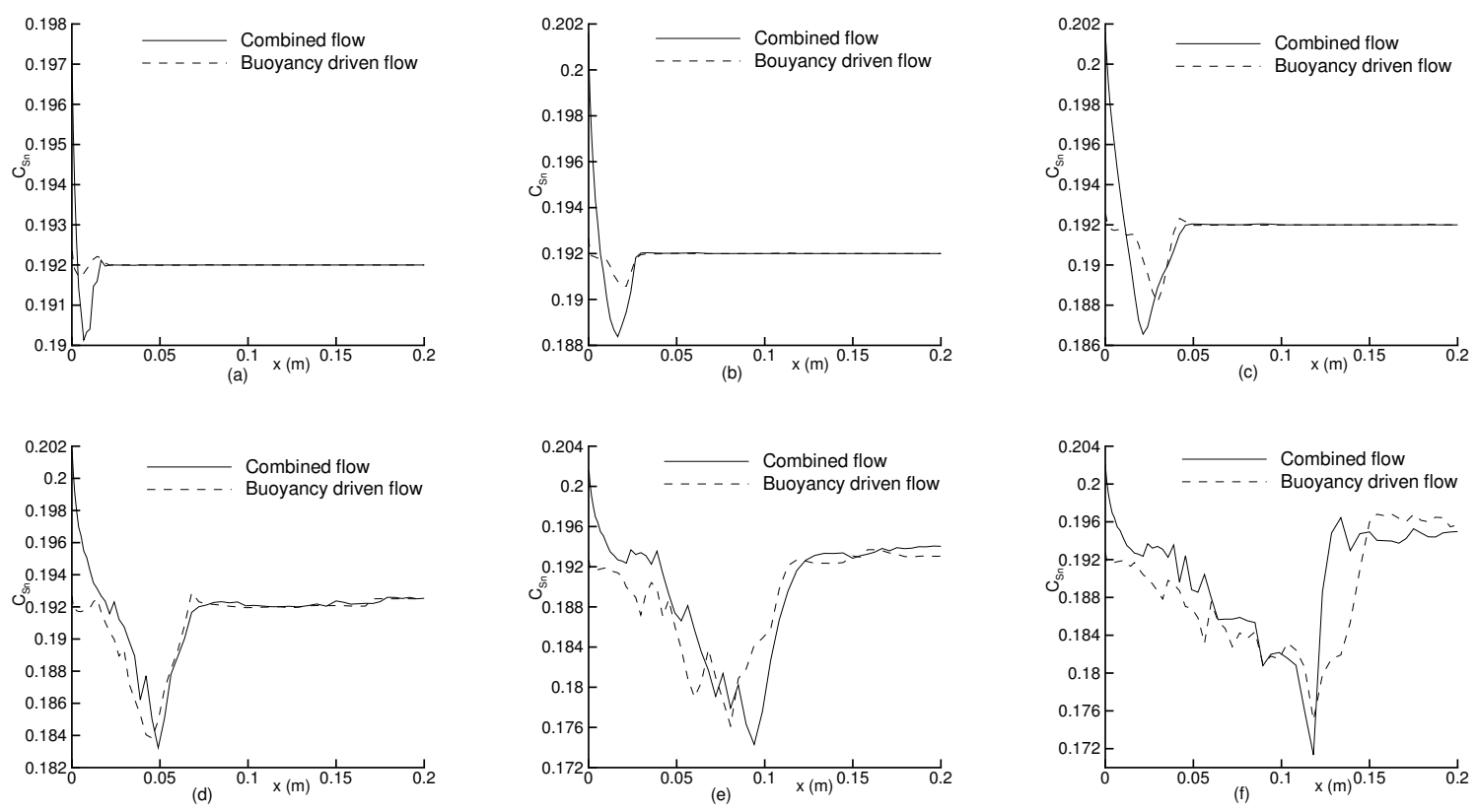

Figure 3. Midplane concentration of Tin (Sn) at different times for Example 1 using grid 2: (a) $25 \mathrm{~s}$, (b) $50 \mathrm{~s}$, (c) $100 \mathrm{~s} \mathrm{(d)} 200 \mathrm{~s} \mathrm{(e)} 400 \mathrm{~s}$ and (f) $600 \mathrm{~s}$.

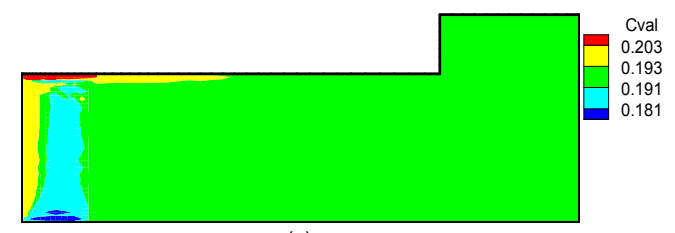

(a)

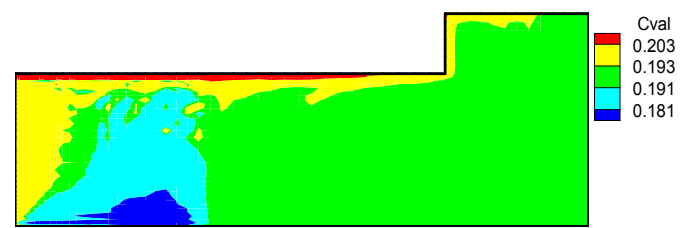

(b)

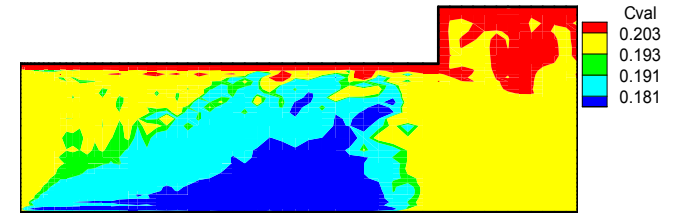

(c)

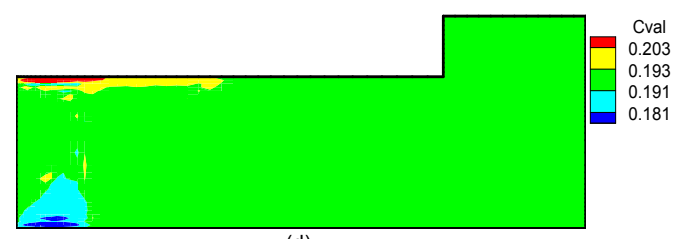

(d)

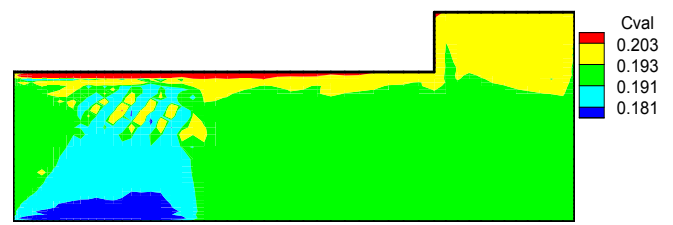

(e)

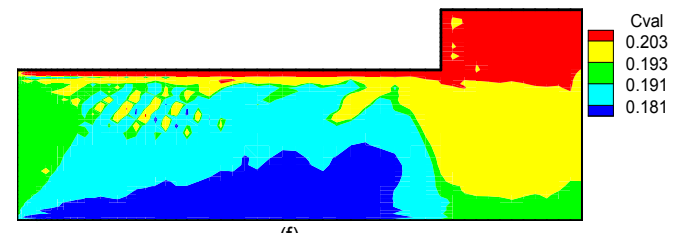

(f)

Figure 4. Distribution of Tin in Example 1 at different times - (a), (b) and (c) correspond to combined flow, (d), (e) and (f) correspond to buoyancy driven flow: (a) and (d) $50 \mathrm{~s}$, (b) and (e) $200 \mathrm{~s}$, (c) and (f) $600 \mathrm{~s}$. 


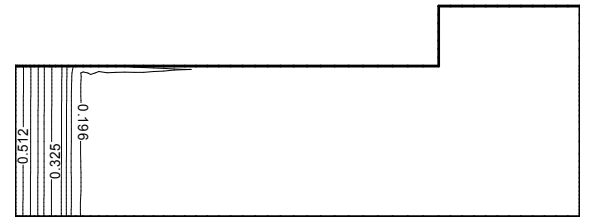

(a)

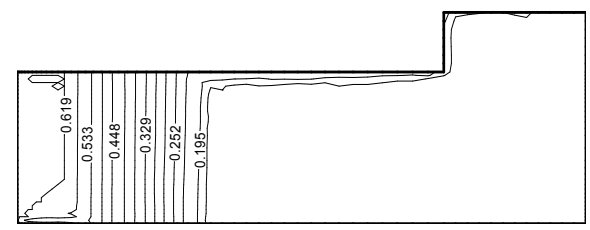

(b)

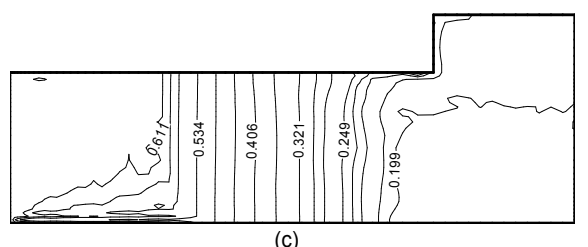

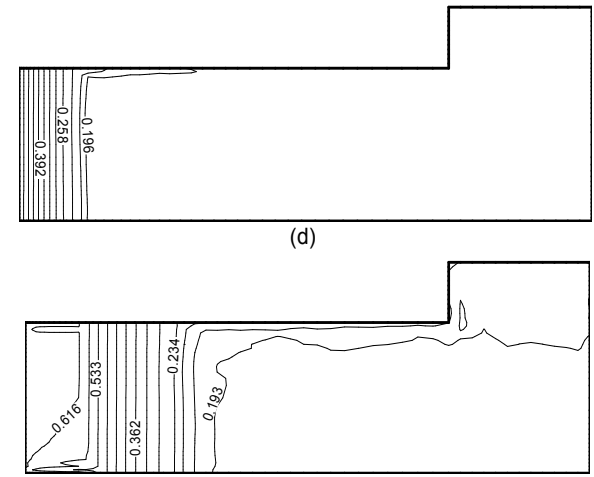

(e)

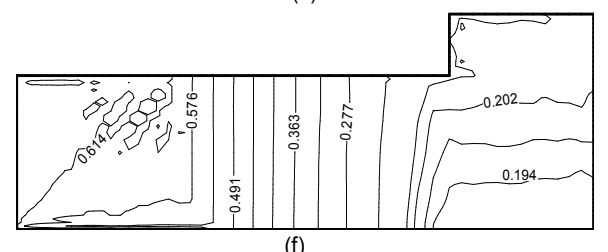

(f)

Figure 5. Liquid solute distribution in Example 1 at different times - (a), (b) and (c) correspond to combined flow, (d), (e) and (f) correspond to buoyancy driven flow: (a) and (d) 50 s, (b) and (e) 200 $\mathrm{s},(\mathrm{c})$ and (f) $600 \mathrm{~s}$.

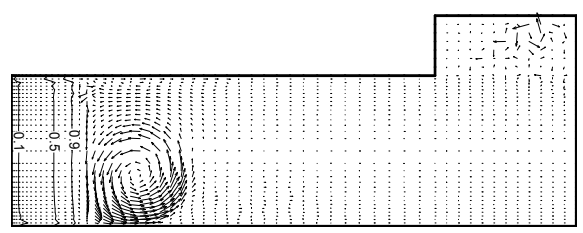

(a)

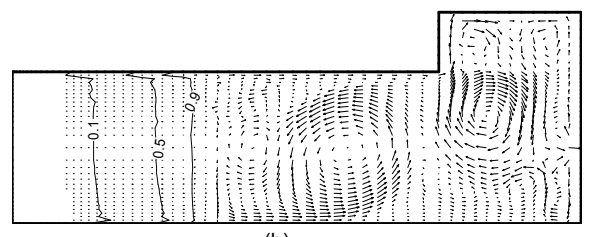

(b)

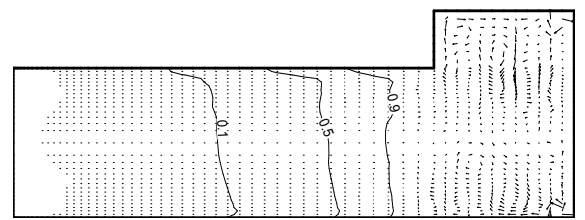

(c)
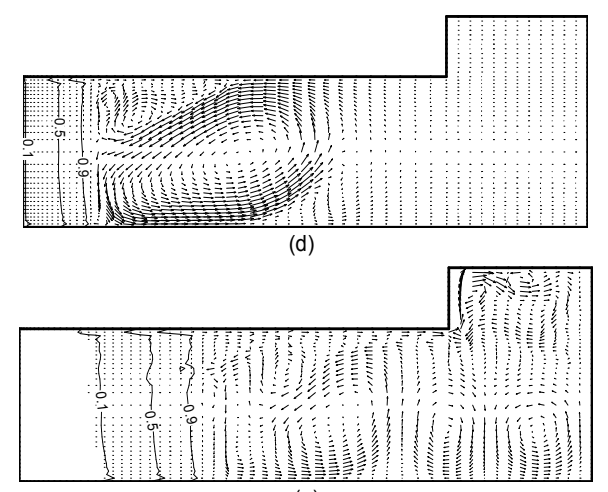

(e)

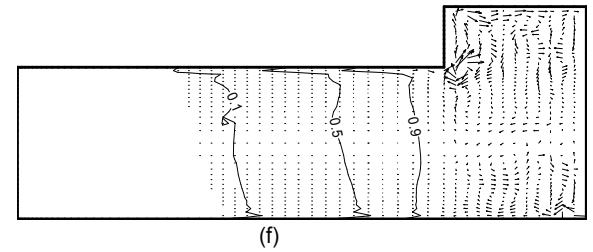

Figure 6. Mass fractions and velocity distribution in Example 1 at different times - (a), (b) and (c) correspond to combined flow, (d), (e) and (f) correspond to buoyancy driven flow: (a) and (d) $50 \mathrm{~s}$, (b) and (e) $200 \mathrm{~s}$, (c) and (f) $600 \mathrm{~s}$. 


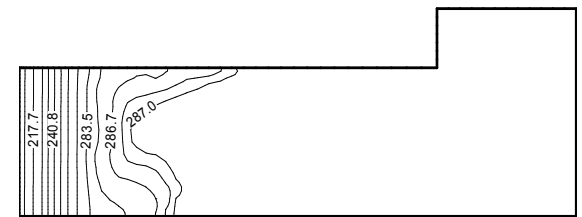

(a)

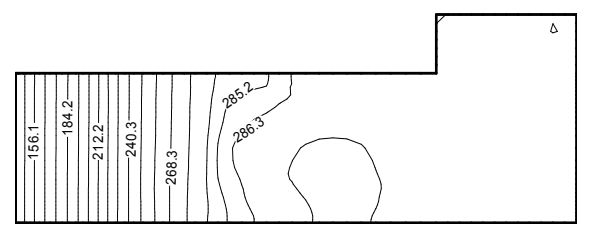

(b)

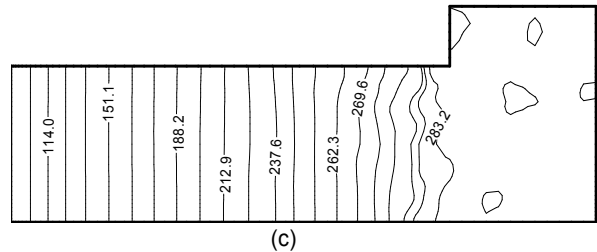

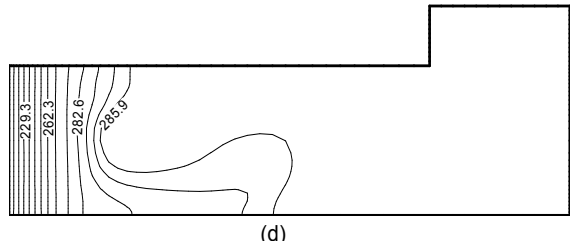

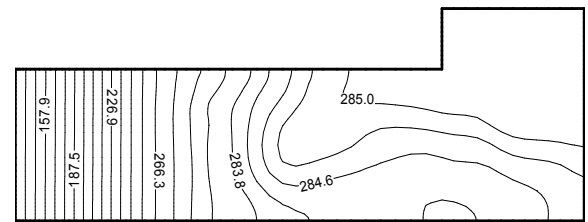

(e)

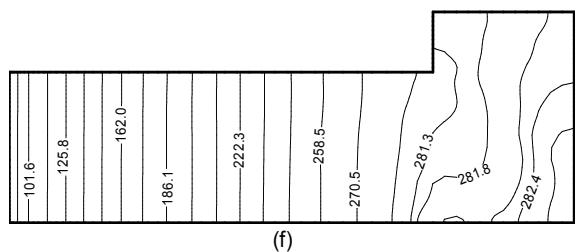

Figure 7. Isotherms in Example 1 at different times - (a), (b) and (c) correspond to combined flow, (d), (e) and (f) correspond to buoyancy driven flow: (a) and (d) $50 \mathrm{~s}$, (b) and (e) $200 \mathrm{~s}$, (c) and (f) $600 \mathrm{~s}$.

gradient of $7700{ }^{\circ} \mathrm{C} / \mathrm{m}$ is applied throughout the domain. The bottom surface is cooled at a constant rate of $0.05{ }^{\circ} \mathrm{C} / \mathrm{s}$. After solidification starts, the thermal gradient is maintained at the top surface. Figures 9 (a)-(e) show the velocity distribution and extent of the mushy zone at different times. Figure 10 shows solute distribution in the cavity at some selected times. As solidification progresses, the velocity field slowly starts to develop. Initially at time $\mathrm{t}=$ 50 seconds (s), the first convection cells form. The mushy zone has developed only until a height of $0.2 \mathrm{~mm}$ similar to that observed in [7]. At $\mathrm{t}=100 \mathrm{~s}$, the velocity field is higher and the resulting convection gives rise to vertical plumes in the center of the cavity. The mushy zone has progressed a distance of $0.8 \mathrm{~mm}$ from the bottom. At $\mathrm{t}=200$ and $300 \mathrm{~s}$, convection increases substantially with progressive solidification and the vertical plumes in the center of the cavity rise higher and become stronger. At $\mathrm{t}=200 \mathrm{~s}$, the mushy zone occupies a distance of $1.4 \mathrm{~mm}$ and at $\mathrm{t}=300 \mathrm{~s}$, a distance of $1.9 \mathrm{~mm}$. These results correlate well with the corresponding quantities observed in [7]. Convection cells continue to grow, weaken, appear and disappear and this contributes to segregation in the cavity. At $t=2000 \mathrm{~s}$, the depth of the mushy zone is approximately $10.6 \mathrm{~mm}$ and the solid shell has formed at the bottom of the cavity. Solute distribution at this time is shown in Fig. 10b. Convection weakens by time $\mathrm{t}=3000 \mathrm{~s}$ and the solid occupies a height of approximately $8.5 \mathrm{~mm}$ from the bottom. Two channels form at both the vertical sidewalls where solute rich liquid penetrates into the mushy zone. The concentration of Tin inside these channels reaches as high as 33.7 wt.\%. Freckles are formed in the interior of the cavity as a consequence of negative segregation caused by fluid flow. Deviation from the initial concentration in these freckles reaches as high as 12.4 wt.\%. The upper half of the cavity is positively segregated. Figure 11 shows the concentration of Tin averaged every $0.5 \mathrm{~mm}$ along the vertical direction. As in the previous example, a 


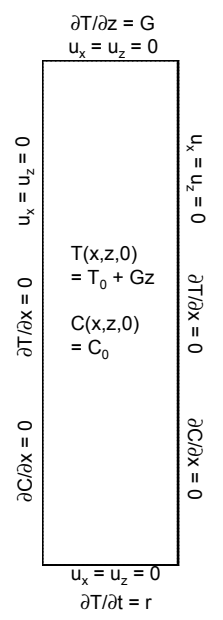

Figure 8. Domain and boundary conditions for vertical solidification of Lead-Tin alloy with anisotropic permeability (Example 2).

zone of negative segregation is found followed by a zone of positive segregation. Table $\mathrm{V}$ gives a comparison between the maximum velocity magnitudes obtained from this simulation and those given in [7], at different times. Some quantitative differences are expected due to a different segregation model used in [7]. The overall CPU time for this problem was 178 hours due to the large total simulation time.

Table V. Comparison of maximum velocity magnitudes between present calculations in Example 2 and those given in [7].

\begin{tabular}{ccc}
\hline Simulation time & $\begin{array}{c}|v|_{\max }(\mathrm{mm} / \mathrm{s}) \\
\text { Present }\end{array}$ & $\begin{array}{c}|v|_{\max }(\mathrm{mm} / \mathrm{s}) \\
\text { From }[7]\end{array}$ \\
\hline 100.0 & 1.78 & 2.10 \\
200.0 & 2.01 & 1.90 \\
300.0 & 1.92 & 1.40 \\
2000.0 & 2.11 & 1.90 \\
3000.0 & 0.94 & 0.6 \\
\hline
\end{tabular}

\subsection{Example 3: Vertical three-dimensional solidification of a Lead-Tin alloy}

Next we consider a three-dimensional example where a Lead-10\% Tin alloy solidifies from the bottom. The length, width and height of the cavity are $0.01 \mathrm{~m}, 0.01 \mathrm{~m}$ and $0.02 \mathrm{~m}$, respectively. The finite element mesh is constructed using 12000 elements with 20 divisions in the $x$ and $y$ axes, and 30 in the $z$-axis. The cooling rate at the bottom is $1.67 \times 10^{-2}{ }^{\circ} \mathrm{C} / \mathrm{s}$ and an initial vertical thermal gradient of $1000{ }^{\circ} \mathrm{C} / \mathrm{m}$ is applied throughout the cavity. No-slip boundary 


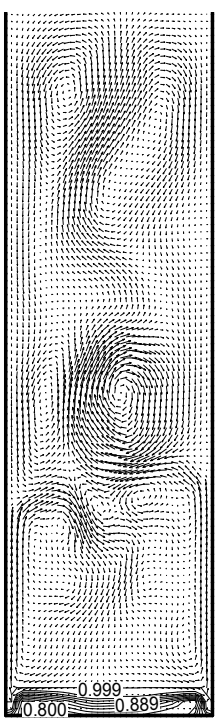

(a)

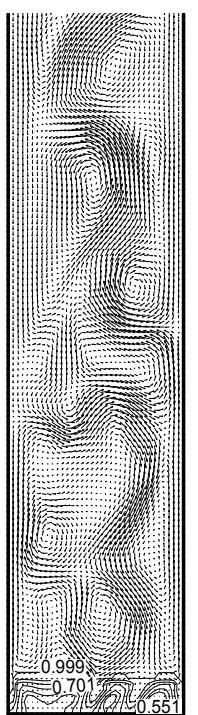

(b)

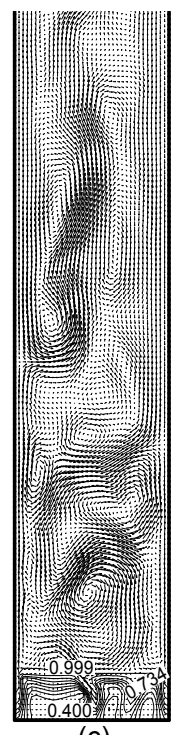

(c)

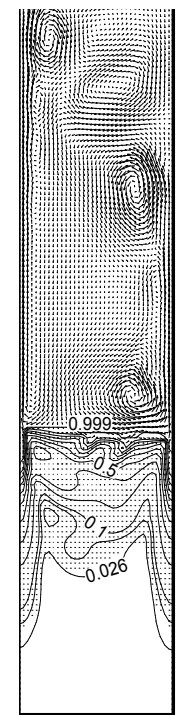

(d)

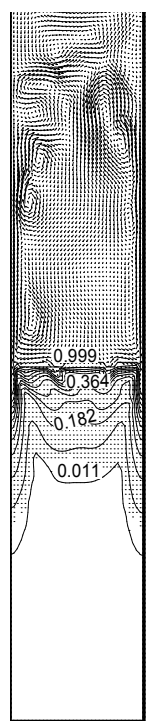

(e)

Figure 9. Velocity field and liquid volume contours for Example 2 at different times: (a) 100 s, (b) 200 s, (c) $300 \mathrm{~s}$, (d) $2000 \mathrm{~s}$ and (e) $3000 \mathrm{~s}$.

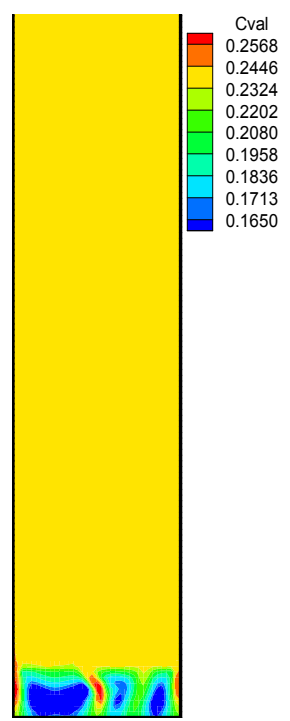

(a)

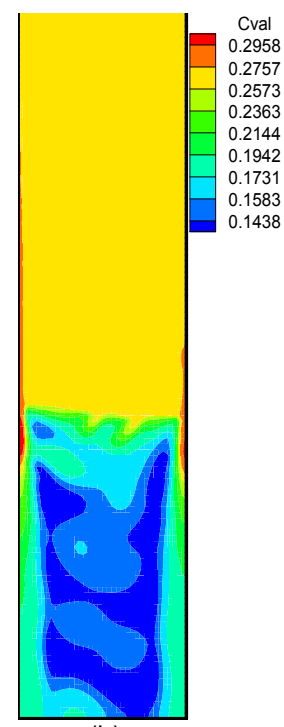

(b)

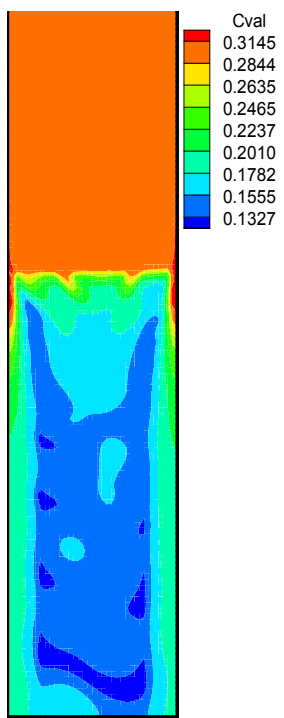

(c)

Figure 10. Solute distribution for Example 2 at different times: (a) 300 s, (b) 2000 s, (c) 3000 s. 


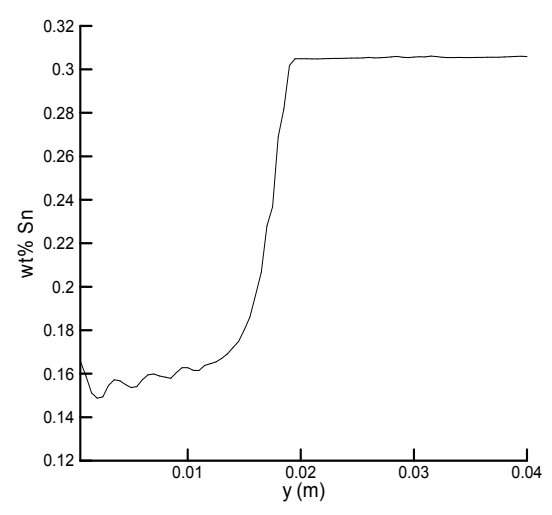

Figure 11. Average concentration of Tin every $0.5 \mathrm{~mm}$ in the vertical direction for Example 2.

Table VI. Comparison of quantities obtained from current calculations for Example 3 and those given in [9].

\begin{tabular}{ccc}
\hline Quantity & Present calculations & From [7] \\
\hline Max. velocity magnitude (mm/s) & 3.81 & 4 \\
Max. concentration (wt \% Sn) & 12.11 & 11.96 \\
Max. number of channels & 9 & 7 \\
\hline
\end{tabular}

conditions are applied on all vertical and horizontal surfaces. The temperature gradient is maintained on the top surface after solidification starts at time $t=0$. Fluid flow in the cavity leads to development of channels with progressive solidification. The simulation is carried until $t=1800 \mathrm{~s}$ and evolution of freckles and channels is studied.

Figures 13 and 14 show the distribution of Tin and liquid volume fraction in the cavity at two different times. Figures 15 (a) and (b) show the concentration profiles of Tin on planes $z=0.005 \mathrm{~m}$ at $t=600 \mathrm{~s}$ and $z=0.015$ at $t=1800 \mathrm{~s}$, respectively. From Figs. 15 (a) and 15 (b) it is clear that more channels are formed here than in the two-dimensional simulation of Example 2. At $t=600 \mathrm{~s}$, nine channels are observed. Greater segregation is observed in channels formed at the edges as against those formed inside the cavity. Table VI gives a comparison between quantities obtained from the present calculation and the work reported in [9] at time $t=600 \mathrm{~s}$. As solidification progresses, solute redistribution occurs throughout the cavity. The main cause of solute redistribution is convection. Greater convective activity is observed in the three-dimensional simulation as against the two-dimensional case of Example 2. At $t=1800 \mathrm{~s}$, the channels on the edges are well developed and concentration of Tin inside these channels reaches as high as $16.1 \mathrm{wt} \%$. Solute depletion occurs near the bottom of the cavity. Localized solute redistribution leads to extensive freckling in the final solidified alloy. This is a major cause of defects and widely varying properties in alloys. The overall CPU time for this problem was 198 hours because of the large total simulation time. The use of matrix free GMRES solver is critical for large scale three-dimensional problems for overcoming memory constraints. 


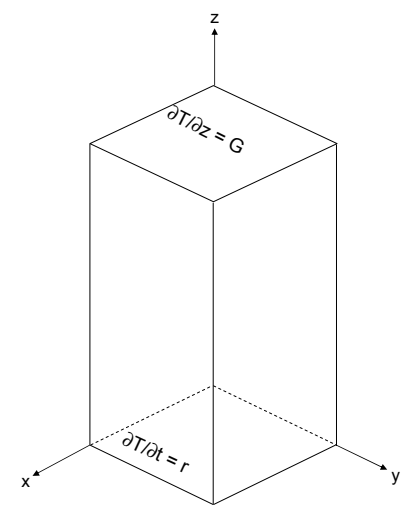

Figure 12. Domain and boundary conditions for vertical solidification of Lead-Tin alloy with anisotropic permeability in three dimensions (Example 3).

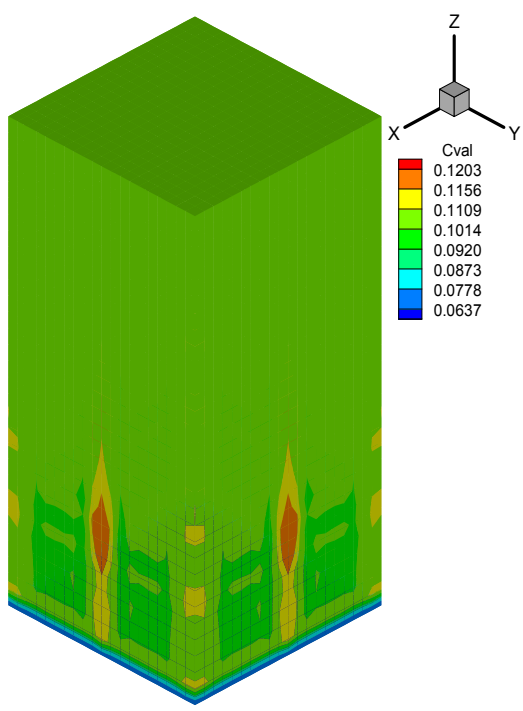

(a)

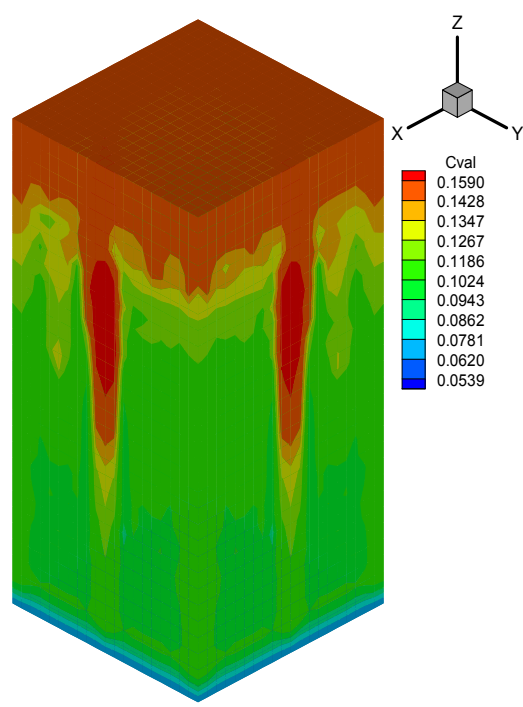

(b)

Figure 13. Macrosegregation profile (distribution of Tin) at two different times for Example 3: (a) 600 s (b) $1800 \mathrm{~s}$.

\section{CONCLUSIONS}

A stabilized FEM formulation, previously developed for porous media and solidification problems was extended to model solidification of alloys with isotropic or anisotropic mushy zone permeability and with different phase densities. Shrinkage driven flow arises due to different densities of the solid and liquid phases. Numerical scheme and solution methodology developed here were first tested for a reference problem involving horizontal solidification of a Lead-Tin alloy where the mushy zone is characterized by an isotropic permeability. Two 


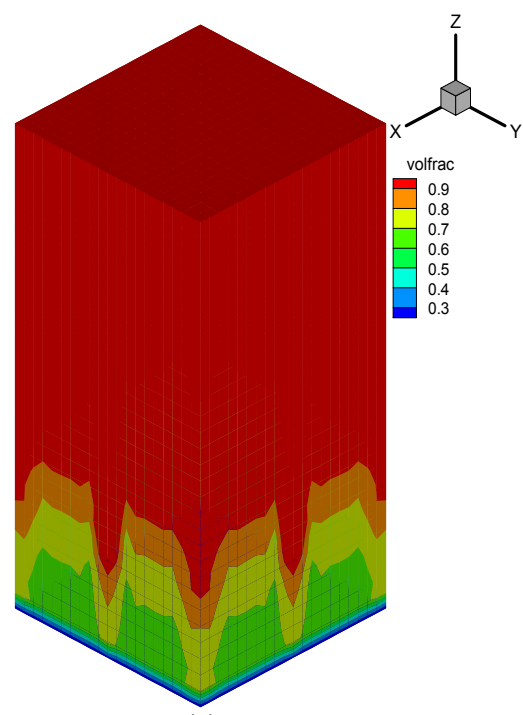

(a)

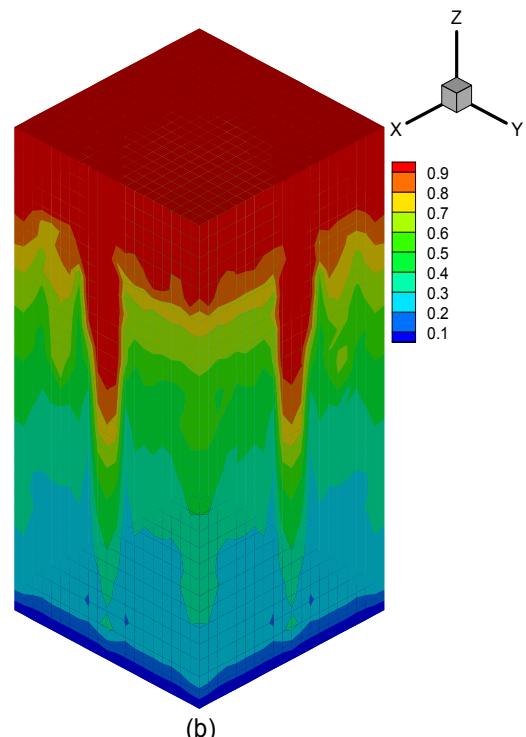

(b)

Figure 14. Liquid volume fractions at two different times for Example 3: (a) $600 \mathrm{~s}$ (b) $1800 \mathrm{~s}$.

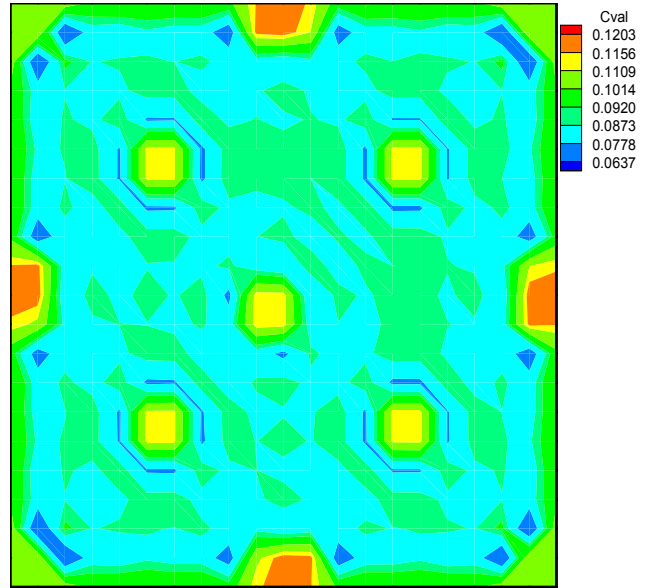

(a)

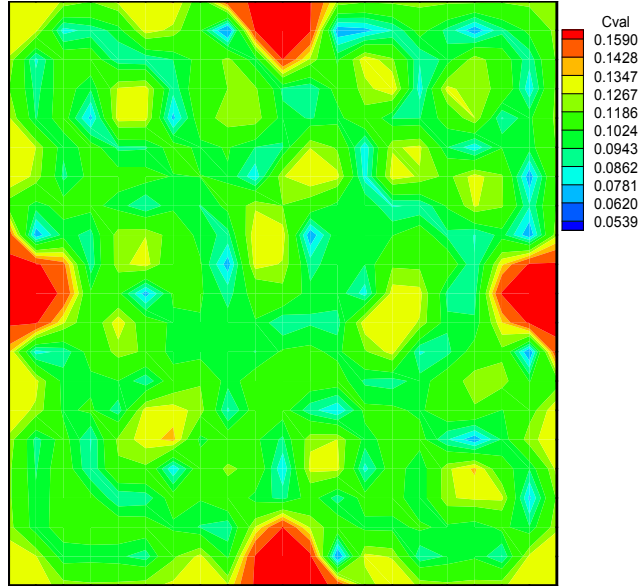

(b)

Figure 15. Macrosegregation profile (distribution of Tin) on $x-y$ plane at different times for Example 3: (a) $z=0.005 \mathrm{~m}, 600 \mathrm{~s}$ (b) $z=0.015 \mathrm{~m}, 1800 \mathrm{~s}$. 
cases were considered - one involving combined shrinkage and buoyancy driven flow and the other involving only buoyancy driven flow. Severe segregation occurs near the top and bottom surfaces for both cases. However, when shrinkage driven flow is present in addition to buoyancy, segregation is magnified near the left cooling wall and near the right bottom. The influence of shrinkage driven flow, arising due to density differences, was found to be greater in regions at or near the solidus temperature. In other regions, segregation was primarily driven by flows arising due to thermal and solutal buoyancy. The algorithm was further validated through a convergence analysis for the same example. Vertical directional solidification of a Lead-Tin alloy was considered next. Here, the mushy zone was characterized by an anisotropic permeability obtained from experimental data and regression analysis. In this case both two-dimensional and three-dimensional examples were attempted. With the mushy zone permeability varying in $x, y$ and $z$ directions, the stabilizing parameters varied correspondingly. Fluid flow here, driven by thermal and solutal buoyancy, led to redistribution of solute in the cavity causing macrosegregation. This led to the development of freckles and channels in the cast alloy. Examples considered here demonstrate the versatility and efficacy of our dimension-independent simulator based on stabilized finite element methods in modeling complex solidification problems. Our current efforts are to extend the model to capture multiscale phenomena and non-equilibrium effects arising during solidification of alloys.

\section{ACKNOWLEDGEMENTS}

The work presented here was funded by the University-Industry Partnerships for Aluminum Industry of the Future Program of the Office of Industrial Technologies of the U.S. Department of Energy (DE-FC07-02ID14396) with additional matching support from Alcoa. This research was conducted using the resources of the Cornell Theory Center which receives support from the New York state and other federal agencies.

\section{REFERENCES}

1. Incropera FP, Bennon WD. A continuum model for momentum, heat and species transport in binary solidliquid phase change systems - I. Model formulation. International Journal of Heat and Mass Transfer 1987; 30: 2161-2170.

2. Bennon WD, Incropera FP. A continuum model for momentum, heat and species transport in binary solid-liquid phase change systems. II. Application to solidification in a rectangular cavity. International Journal of Heat and Mass Transfer 1987; 30: 2171-2187.

3. Voller VR, Brent AD, Prakash C. The modeling of heat, mass and solute transport in solidification systems. International Journal of Heat and Mass Transfer 1989; 32: 1718-1731.

4. Xu D, Li P. Numerical method for solution of strongly coupled binary alloy solidification problems. Numerical Heat Transfer A 1991; 20: 181-201.

5. Xu D, Li P. Gravity and solidification shrinkage induced liquid flow in a horizontally solidified alloy ingot. Numerical Heat Transfer A 1991; 20: 203-221.

6. Felicilli SD, Heinrich JC, Poirier DR. Finite element analysis of directional solidification of multicomponent alloys. International Journal for Numerical Methods in Fluids, 27 (1998), 207-227.

7. Heinrich JC, Poirier DR, Convection modeling in directional solidification. C.R.Mechanique (2004); 332: 429-445.

8. Mcbridge E, Heinrich JC, Poirier DR. Numerical simulation of incompressible flow driven by density variations during phase change. International Journal for Numerical Methods in Fluids 1999; 31: 787800. 
9. Felicilli SD, Heinrich JC, Poirier DR. Three dimensional simulations of freckles in binary alloys. Journal of Cyrstal Growth 1998; 191 : 879-888.

10. Krane M J M, Incropera FP. Analysis of the effect of shrinkage on macrosegregation in alloy solidification. Transport Phenomenon in Solidification, ASME HTD-Vol. 284/AMD Vol. 182, ASME, New York, 1994, 13-27.

11. Zabaras N, Samanta D. A stabilized volume-averaging finite element method for flow in porous media and binary alloy solidification processes. International Journal for Numerical Methods in Engineering 2004; 60(6):1103-1138.

12. Shyy W, Udaykumar HS, Rao MM, Smith RW. Computational Fluid Dynamics with Moving Boundaries (1st edn). Taylor and Francis: Washington, D.C., 1996.

13. Ni J, Beckermann C. A volume-averaged two-phase model for transport phenomena during solidification. Metallurgical Transactions B 1990; 22B: 349-361.

14. Felicilli SD, Heinrich JC, Poirier DR. Numerical model for dendritic solidification of binary alloys, Numerical Heat Transfer B 1993; 23: 461-481.

15. Masud A, Hughes TJR. A stabilized mixed finite element method for Darcy flow. Computer Methods in Applied Mechanics and Engineering 2002; 191: 4341-4370.

16. Tezduyar TE, Mittal S, Ray SE, Shih R. Incompressible flow computations with stabilized bilinear and linear equal-order-interpolation velocity pressure elements. Computer Methods in Applied Mechanics and Engineering 1992; 95: 221-242.

17. Tezduyar TE. Stabilized finite element formulations for incompressible flow computations. Advances in Applied Mechanics 1992; 28: 1-43.

18. Tezduyar TE, Osawa Y. Finite element stabilization parameters computed from element matrices and vectors. Computer Methods in Applied Mechanics and Engineering 2000; 190: 411-430.

19. Badri Narayanan VA, Zabaras N. Variational multiscale stabilized FEM formulations for transport equations: stochastic advection-diffusion and incompressible stochastic Navier-Stokes equations. Journal of Computational Physics 2005; 202/1: 94-133.

Copyright (C) 2005 John Wiley \& Sons, Ltd.

Int. J. Numer. Meth. Engng 2005; :1-28

Prepared using nmeauth.cls 